\title{
ACT/FHS System Identification Including Rotor and Engine Dynamics
}

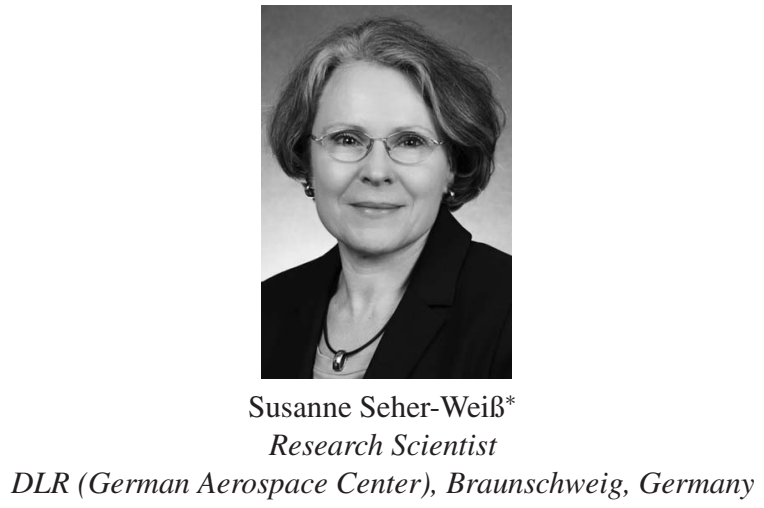

\begin{abstract}
At the German Aerospace Center (DLR) Institute of Flight Systems, models of the Active Control Technology/Flying Helicopter Simulator (ACT/FHS), an EC135 with a fly-by-wire/light flight control system, are needed for control law development and simulation. Thus, models are sought that cover the whole flight envelope and are valid over a broad range of frequencies. Furthermore, if the models are to be used in the feedforward loop of the model following the control system, they have to be invertible and thus should not have any positive transmission zeros. For rotor flapping, the explicit formulation with flapping angles was modified slightly to avoid positive transmission zeros. For the regressive lead-lag, a simple model formulation was found that needs only one dipole with two states. The engine dynamics were first modeled separately and then coupled to the body/rotor model. The final integrated model has 17 states and yields a good match for frequencies up to $30 \mathrm{rad} / \mathrm{s}$. All system identification was performed using the maximum likelihood method in the frequency domain.
\end{abstract}

\section{Nomenclature}

$\begin{array}{ll}\boldsymbol{A}, \boldsymbol{B}, \boldsymbol{C}, \boldsymbol{D} & \text { state-space representation of dynamic model } \\ a_{x}, a_{y}, a_{z} & \text { body-fixed linear accelerations, } \mathrm{m} / \mathrm{s}^{2} \\ C_{L_{\alpha}} & \text { blade lift curve slope, } 1 / \mathrm{rad} \\ C_{T} & \text { thrust coefficient, }=T /\left[\rho \pi R^{2}(\Omega R)^{2}\right] \\ C_{0} & \text { inflow constant } \\ c & \text { rotor blade chord, } \mathrm{m} \\ D_{\delta_{\text {lon }}}, D_{\delta_{\text {lat }}} & \text { control derivatives of the lead-lag dipole } \\ E_{. .} & \text {engine model parameters } \\ e & \text { hinge offset, } \mathrm{m} \\ g & \text { acceleration of gravity, } \mathrm{m} / \mathrm{s}^{2} \\ \boldsymbol{H} & \text { transfer function matrix } \\ \boldsymbol{I} & \text { identity matrix } \\ I_{\beta} & \text { blade flapping moment of inertia, } \mathrm{kg} \mathrm{m}{ }^{2} \\ K_{\beta} & \text { flapping stiffness, Nm/rad } \\ K_{\theta_{0}} & \text { control gain, rad } / \% \\ L_{. .}, M . ., N . . & \text { moment derivatives } \\ L f . ., M f . . & \text { flapping moment derivatives } \\ m & \text { aircraft mass, kg } \\ p, q, r & \text { roll, pitch, and yaw rates, rad } / \mathrm{s}\end{array}$

*Corresponding author; email: susanne.seher-weiss@dlr.de.

Presented, in part, at the AHS International 73rd Annual Forum \& Technology Display, Fort Worth, TX, May 9-11, 2017. Manuscript received June 2017; accepted January 2019.

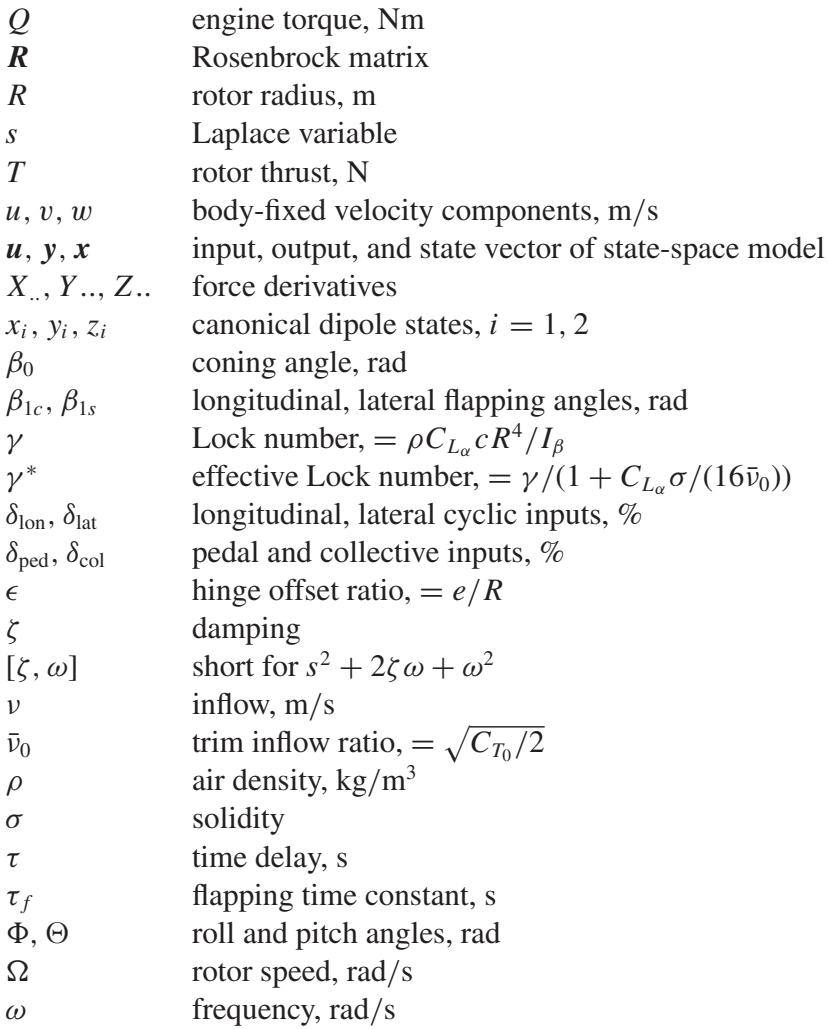




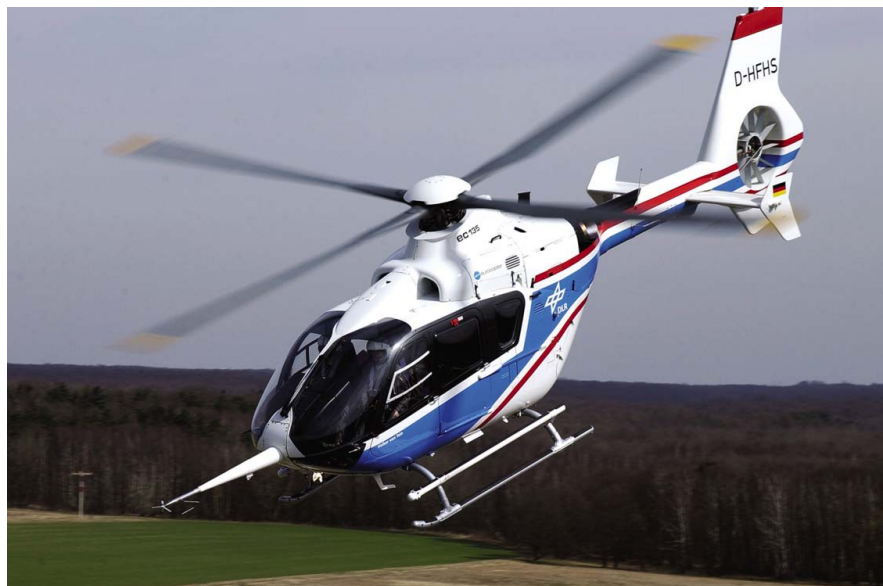

Fig. 1. DLR research helicopter ACT/FHS.

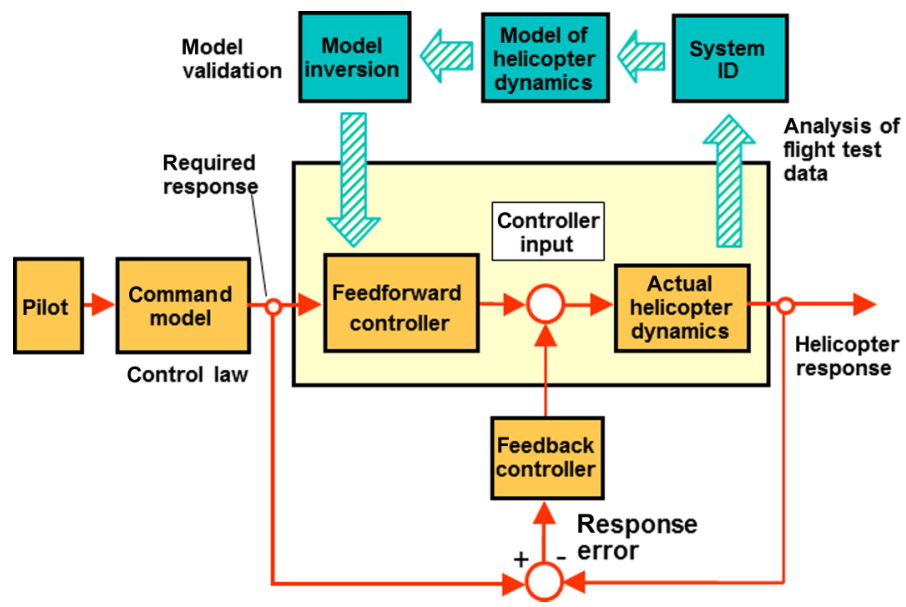

Fig. 2. MFCS general structure.

\section{Subscripts \\ 0 \\ en \\ 11 \\ engine \\ lead-lag}

drops rank (see Refs. 10,11). The transmission zeros are calculated as the eigenvalues of the Rosenbrock matrix

$$
\boldsymbol{R}(s)=\left[\begin{array}{cc}
s \boldsymbol{I}-\boldsymbol{A} & -\boldsymbol{B} \\
\boldsymbol{C} & \boldsymbol{D}
\end{array}\right]
$$

It can be shown that the transmission zeros are equal to the poles of the inverted system. In general, the transmission zeros of a multiinput/multioutput (MIMO) system do not coincide with zeros of the transfer function matrix.

The identified model that is to be used in the feedforward controller of the MFCS should not have any positive transmission zeros because positive transmission zeros would result in unstable poles in the inverted model. The transmission zeros depend on the output variables that are chosen. In case of the ACT/FHS control system, these are the three angular rates and the vertical acceleration $\left(p, q, r, a_{z}\right)$.

Theoretically, inner-outer factorization could be used to construct a stable inverse of a nonminimum phase model (a model with positive transmission zeros). This system inversion procedure is costly and may lead to different model structures for the inverted systems at the five operating points. In such a case, interpolation between the operating points requires fine-tuning by hand, which means that this type of system inversion cannot be included in an automatic control system generation process. Therefore, models without positive transmission zeros are highly preferred as long as the degradation compared to a minimum phase model is small enough.

Tischler states in chapter 11 of Ref. 12 that a model to be used for flight control development should be accurate over the frequency range of $0.3 \omega_{\text {co }}$ to $3 \omega_{\text {co }}$ where $\omega_{\text {co }}$ is the crossover frequency. For the ACT/FHS with a cutoff frequency of $3 \mathrm{rad} / \mathrm{s}$ in pitch and $5 \mathrm{rad} / \mathrm{s}$ in roll, this would mean that the models should accurately cover the frequency range of $1-15 \mathrm{rad} / \mathrm{s}$. Experience has shown that this frequency range is too narrow and has to be extended at the lower end for flying with external loads and at the upper end to avoid exciting structural modes. The aim was thus to develop models that are accurate over the frequency range of $0.5-30 \mathrm{rad} / \mathrm{s}$. This means that an extended model structure is necessary that explicitly includes the regressive flapping, coupled inflow/coning, and regressive lead-lag states of the rotor.

In the past, different modeling aspects of ACT/FHS system identification have been described in separate papers (see Refs. 13,14). In this paper, these results are reevaluated with respect to the requirements of model interpolation and model inversion. Furthermore, results from investigations using the optimized predictor-based subspace identification

$$
\boldsymbol{H}(s)=\boldsymbol{C}(s \boldsymbol{I}-\boldsymbol{A})^{-1} \boldsymbol{B}+\boldsymbol{D}
$$


(PBSIDopt) method as shown in Ref. 15 are used to develop a simplified formulation for the influence of the regressive lead-lag. Also, this paper shows how deficits in yaw rate approximation are reduced by engine modeling.

The paper will first describe the modeling that was used for the different rotor degrees of freedom (DOF). Next, the development of a dynamic engine model and its integration into the overall model will be shown. Finally, some results for the fully integrated model will be presented.

\section{Flapping}

Rotor flapping is usually accounted for in system identification modeling with the explicit formulation developed by Tischler (chapter 15 in Ref. 12). This explicit formulation includes two coupled first-order differential equations for the longitudinal and lateral flapping angles $\beta_{1 c}$ and $\beta_{1 s}$ that are triggered by the longitudinal and lateral cyclic control inputs:

$$
\begin{gathered}
\tau_{f} \dot{\beta}_{1 s}=-\beta_{1 s}+L f_{\beta_{1 c}} \beta_{1 c}+\tau_{f} p+L f_{\delta_{\text {lon }}} \delta_{\text {lon }}+L f_{\delta_{\text {lat }}} \delta_{\text {lat }} \\
\tau_{f} \dot{\beta}_{1 c}=-\beta_{1 c}+M f_{\beta_{1 s}} \beta_{1 s}+\tau_{f} q+M f_{\delta_{\text {lon }}} \delta_{\text {lon }}+M f_{\delta_{\text {lat }}} \delta_{\text {lat }}
\end{gathered}
$$

The flapping angles are coupled to the rigid-body equations via

$$
\begin{aligned}
\dot{u}= & X_{u} u+X_{v} v+X_{w} w-w_{0} q+\left(X_{r}+v_{0}\right) r-g \cos \Theta_{0} \Theta \\
& +X_{\beta_{1 c}} \beta_{1 c}+X_{\delta_{\mathrm{col}}} \delta_{\mathrm{col}}+X_{\delta_{\mathrm{ped}}} \delta_{\mathrm{ped}} \\
\dot{v}= & Y_{u} u+Y_{v} v+Y_{w} w+\left(Y_{p}+w_{0}\right) p+\left(Y_{r}-u_{0}\right) r+g \cos \Theta_{0} \Phi \\
& +Y_{\beta_{1 s}} \beta_{1 s}+Y_{\delta_{\mathrm{col}}} \delta_{\mathrm{col}}+Y_{\delta_{\mathrm{ped}}} \delta_{\mathrm{ped}} \\
\dot{p}= & L_{u} u+L_{v} v+L_{w} w+L_{r} r+L_{\beta_{1 s}} \beta_{1 s}+L_{\delta_{\mathrm{col}}} \delta_{\mathrm{col}}+L_{\delta_{\mathrm{ped}}} \delta_{\mathrm{ped}} \\
\dot{q}= & M_{u} u+M_{v} v+M_{w} w+M_{r} r+M_{\beta_{1 c}} \beta_{1 c}+M_{\delta_{\mathrm{col}}} \delta_{\mathrm{col}}+M_{\delta_{\mathrm{ped}}} \delta_{\mathrm{ped}}
\end{aligned}
$$

Compared to a standard six-DOF model, the cyclic control derivatives $X_{\delta_{\text {lon }}}, X_{\delta_{\text {lat }}}, Y_{\delta_{\text {lon }}}, Y_{\delta_{\text {lat }}}, L_{\delta_{\text {lon }}}, L_{\delta_{\text {lat }}}, M_{\delta_{\text {lon }}}$, and $M_{\delta_{\text {lat }}}$ have been replaced by the corresponding control derivatives $L f_{\delta_{\text {lon }}}, L f_{\delta_{\text {lat }}}, M f_{\delta_{\text {lon }}}$, and $M f_{\delta_{\text {lat }}}$ in the equations of the flapping dynamics. Similarly, the standard force and moment derivatives $X_{p}, X_{q}, Y_{q}, L_{p}, L_{q}, M_{p}$, and $M_{q}$ have been replaced by the rotor force and moment terms $L_{\beta_{1 s}}, M_{\beta_{1 c}}, X_{\beta_{1 c}}$, and $Y_{\beta_{1 s}}$ that couple the main rotor to the fuselage. The quasi-static force derivative $Y_{p}$ is often retained to account for tail rotor effects.

The force terms $X_{\beta_{1 c}}$ and $Y_{\beta_{1 s}}$ are constrained due to physical considerations $Y_{\beta_{1 s}}=-X_{\beta_{1 c}}$. Their numerical value is theoretically equal to the acceleration of gravity $g$ and therefore often constrained to this value for the identification:

$$
X_{\beta_{1 c}}=-Y_{\beta_{1 s}}=g
$$

A theoretical value for the rotor flap time constant $\tau_{f}$ can be calculated from the hinge offset $e$ and the effective lock number $\gamma^{*}$,

$$
\frac{1}{\tau_{f}}=\frac{\gamma^{*} \Omega}{16}\left(1-\frac{8 e}{3 R}\right)
$$

but $\tau_{f}$ is usually left as a free parameter in the identification.

For the identification of the ACT/FHS, an implicit formulation of the flapping equations as first described in Ref. 4 had been used. Whereas the explicit formulation from Eqs. (4) contains the flapping angles explicitly as state variables, the implicit formulation inserts the flapping angles into the equations for pitch an droll rate, resulting in $\dot{p}$ and $\dot{q}$ as two additional state variables:

$$
\begin{aligned}
& \ddot{p}=\hat{L}_{p} p+\hat{L}_{\dot{p}} \dot{p}+\hat{L}_{\delta_{\text {lat }}} \delta_{\text {lat }} \\
& \ddot{q}=\hat{M}_{q} q+\hat{M}_{\dot{q}} \dot{q}+\hat{M}_{\delta_{\text {lon }}} \delta_{\text {lon }}
\end{aligned}
$$

In Ref. 13, it was shown that the explicit and the implicit formulation of the rotor flapping dynamics produces equivalent results. The trade-off between implicit and explicit modeling of the flapping dynamics in the context of rotor state feedback is discussed in Ref. 16.

The reason for using this implicit formulation was that for the ACT/FHS the explicit formulation led to models with positive transmission zeros and that thus the implicit formulation was preferred for model following control.

On the other hand, it was expected that models with explicit flapping would be superior regarding interpolation between different reference speeds for model stitching (see Ref. 5) due to better separation of the rigid-body and rotor DOF. Thus, modification of the explicit model formulation was attempted in order to solve the problem with the positive transmission zeros.

Let $\mathbf{A}_{\mathrm{ex}}$ and $\mathbf{B}_{\mathrm{ex}}$ be the system matrices of the explicit model

$$
\dot{\mathbf{x}}_{\mathrm{ex}}=\mathbf{A}_{\mathrm{ex}} \mathbf{x}_{\mathrm{ex}}+\mathbf{B}_{\mathrm{ex}} \mathbf{u}
$$

with a state vector of

$$
\mathbf{x}_{\mathrm{ex}}^{T}=\left[u, v, w, p, q, r, \Phi, \Theta, \beta_{1 s}, \beta_{1 c}\right]
$$

and control inputs $\mathbf{u}$. Then a transformation matrix $\mathbf{T}$ that consists of an $8 \times 8$ identity matrix $\mathbf{I}_{8}$, an $8 \times 2$ matrix of zeros $\mathbf{O}_{8,2}$, and the rows of $\mathbf{A}_{\text {ex }}$ that correspond to $q$ and $p$ :

$$
\mathbf{T}=\left[\begin{array}{cc}
\mathbf{I}_{8} & \mathbf{O}_{8,2} \\
\mathbf{A}_{\mathrm{ex}}(5,1: 8) & \mathbf{A}_{\mathrm{ex}}(5,9: 10) \\
\mathbf{A}_{\mathrm{ex}}(4,1: 8) & \mathbf{A}_{\mathrm{ex}}(4,9: 10)
\end{array}\right]
$$

can be used to transform the system from Eq. (9) via

$$
\mathbf{A}_{\text {im }}=\mathbf{T} \mathbf{A}_{\mathrm{ex}} \mathbf{T}^{-1}, \quad \mathbf{B}_{\mathrm{im}}=\mathbf{T} \mathbf{B}_{\mathrm{ex}}
$$

into an implicit model

$$
\dot{\mathbf{x}}_{\mathrm{im}}=\mathbf{A}_{\mathrm{im}} \mathbf{x}_{\mathrm{im}}+\mathbf{B}_{\mathrm{im}} \mathbf{u}
$$

with a state vector of

$$
\mathbf{x}_{\mathrm{im}}^{T}=[u, v, w, p, q, r, \Phi, \Theta, \dot{q}, \dot{p}]
$$

Transforming the models identified with the explicit flapping equations into the implicit formulation as just described and comparing the resulting matrices showed that the main differences were in the collective and pedal control derivatives. Therefore, the explicit formulation of rotor flapping was modified in such a way that the collective control input also acts on the flapping angles:

$$
\begin{gathered}
\tau_{f} \dot{\beta}_{1 s}=-\beta_{1 s}+L f_{\beta_{1 c}} \beta_{1 c}+\tau_{f} p+L f_{\delta_{\text {lon }}} \delta_{\text {lon }}+L f_{\delta_{\text {lat }}} \delta_{\text {lat }}+L f_{\delta_{\text {col }}} \delta_{\text {col }} \\
\tau_{f} \dot{\beta}_{1 c}=-\beta_{1 c}+M f_{\beta_{1 s}} \beta_{1 s}+\tau_{f} q+M f_{\delta_{\text {lon }}} \delta_{\text {lon }}+M f_{\delta_{\text {lat }}} \delta_{\text {lat }}+M f_{\delta_{\text {col }}} \delta_{\text {col }}
\end{gathered}
$$

The corresponding quasi-static collective control derivatives $L_{\delta_{0}}$ and $M_{\delta_{0}}$ in the pitch and roll rate equations were dropped. This slight model modification led to models for the ACT/FHS without positive transmission zeros. The quasi-static pedal control derivatives could remain unchanged.

The match with this modified explicit flapping formulation is almost identical to the match achieved with the models with the implicit flapping formulation as can be seen from Fig. 3 .

Thus reformulating the explicit flapping so that the flapping angles are also excited by the collective control input yields a model that provides a 

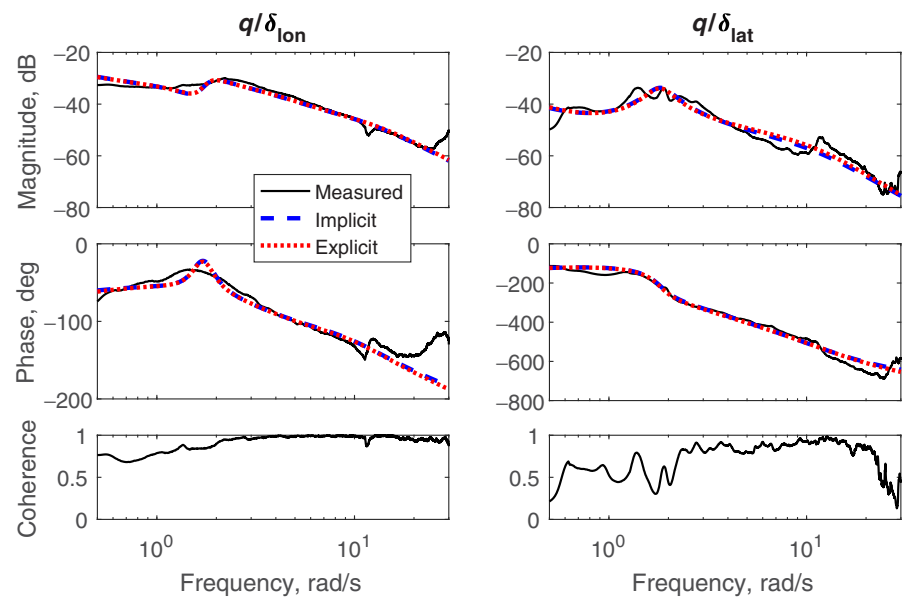

Fig. 3. Match in the pitch rate for implicit and modified explicit flapping $(60 \mathrm{kt})$.

good separation of rigid-body and rotor parameters, has no positive transmission zeros, and provides a match that is comparable to the implicit flapping formulation.

\section{Inflow/Coning}

Modeling of the inflow/coning dynamics is necessary to capture the rising amplitude in the frequency response for vertical acceleration due to collective input and can be achieved by different modeling approaches.

The approach most widely used for modeling the inflow/coning dynamics and their coupling to the fuselage is the hybrid formulation developed by Tischler (chapter 15 in Ref. 12). It is based on the work by Chen and Hindson (Ref. 17) who developed analytical models for the coupled inflow/coning/heave dynamics.

The first-order inflow dynamics are written as

$$
\begin{aligned}
\dot{v}= & \frac{-75 \pi \Omega}{32}\left(\bar{v}_{0}+\frac{C_{L \alpha} \sigma}{16}\right) C_{0} v+v_{\dot{\beta}} \dot{\beta}_{0} \\
& +\frac{25 \pi \Omega^{2} R}{32}\left(\frac{C_{L \alpha} \sigma}{8}\right) C_{0} K_{\theta_{0}} \delta_{\mathrm{col}}
\end{aligned}
$$

with the trim inflow ratio $\bar{v}_{0}$ and thrust coefficient $C_{T_{0}}$ using $T_{0}=m g$ for the trim thrust. The control gain $K_{\theta_{0}}$ transforms collective input to effective blade root pitch angle $\left(\theta_{0}\right)$.

For hovering flight, an analytical expression is available for the $v_{\dot{\beta}}$ coefficient:

$$
v_{\dot{\beta}}=\frac{-25 \pi \Omega R}{32}\left(\bar{v}_{0}+\frac{C_{L \alpha} \sigma}{8}\right) C_{0}
$$

The rigid-blade coning dynamics, ignoring the influence of hinge offset and flapping spring, are expressed as a second-order differential equation:

$$
\ddot{\beta}_{0}=-\frac{\Omega \gamma}{8} \dot{\beta}_{0}-\Omega^{2} \beta_{0}-\frac{\Omega \gamma}{6 R} v+\frac{\Omega^{2} \gamma}{8} K_{\theta_{0}} \delta_{\mathrm{col}}
$$

resulting in two states, coning angle $\beta_{0}$ and coning rate $\dot{\beta}_{0}$.

Finally, the coning/inflow dynamics are coupled to the fuselage through the thrust coefficient $C_{T}$, to achieve the hybrid model structure

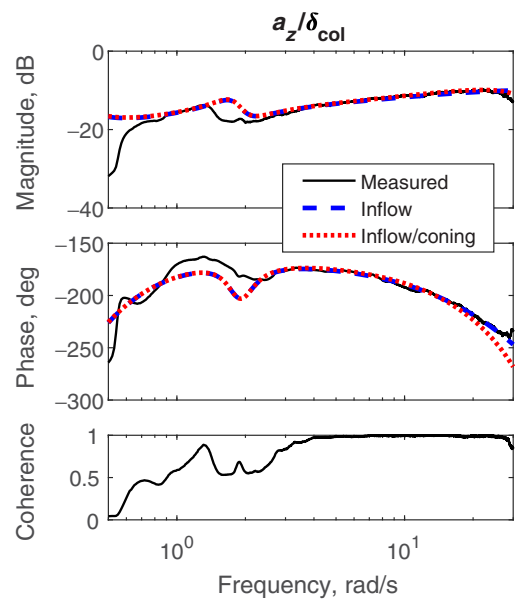

Fig. 4. Match in vertical acceleration for models with and without coning $(60 \mathrm{kt})$.

for the vertical dynamics

$$
\begin{aligned}
\dot{w}= & Z_{u} u+Z_{v} v+Z_{w} w+\left(Z_{p}-v_{0}\right) p+\left(Z_{q}+u_{0}\right) q+Z_{r} r \\
& -g \cos \Phi_{0} \sin \Theta_{0} \Theta-\frac{\rho \pi R^{2}(\Omega R)^{2}}{m} C_{T}+Z_{\delta_{\text {lon }}} \delta_{\text {lon }} \\
& +Z_{\delta_{\text {lat }}} \delta_{\text {lat }}+Z_{\delta_{\text {ped }}} \delta_{\text {ped }}
\end{aligned}
$$

where the perturbation thrust coefficient $C_{T}$ is given by

$$
C_{T}=\frac{0.543}{\Omega^{2} R} \frac{1}{C_{0}} \dot{v}+\frac{4 \bar{\nu}_{0}}{\Omega R} v+\frac{4 \bar{\nu}_{0}}{3 \Omega} \dot{\beta}_{0}
$$

Most system identification performed using the hybrid formulation uses the Carpenter-Fridovich model, which means setting the inflow time constant to $C_{0}=0.639$ (see Ref. 18). Owing to the lack of blade motion and inflow and thrust measurements, all derivatives of the inflow and coning equations are usually fixed at their theoretical predictions and only $Z_{w}$ is estimated.

The hybrid inflow/coning model was applied to ACT/FHS flight-test data in Ref. 14. Owing to its bearingless design, the rotor of the ACT/FHS has a relatively large equivalent hinge offset of $10 \%$. Therefore, the coning equation (18) had to be extended to include the influence of hinge offset.

However, when analyzing the identified models, it was discovered that the inclusion of the coning motion led to a high-frequency positive transmission zero for the forward flight cases, thus making it difficult to use these models as a basis for the feedforward controller. As can be seen in Fig. 4, the inclusion of coning does not really improve the match of $a_{z} / \delta_{\text {col }}$ in forward flight. Therefore, it was decided to drop the coning equations by setting the coefficients corresponding to $\dot{\beta}_{0}$ to zero in Eqs. (16) and (20).

As it was desired to have the same model structure for all speeds to allow for model stitching, the coning equations were also dropped for the hover case even though this leads to a slight degradation in the match of the frequency response of vertical acceleration due to collective input for frequencies above $12 \mathrm{rad} / \mathrm{s}$ (see Fig. 5).

It has to be stated that the models without positive transmission zeros still exhibit positive zeros in the single input/single output (SISO) transfer functions of heave axis $\left(w / \delta_{\text {col }}\right)$.

Positive transmission zeros pose no problem if the identified models are used for purposes where they do not have to be inverted (e.g., simulation). Therefore, models that include the coning motion were identified in addition to those without coning. 


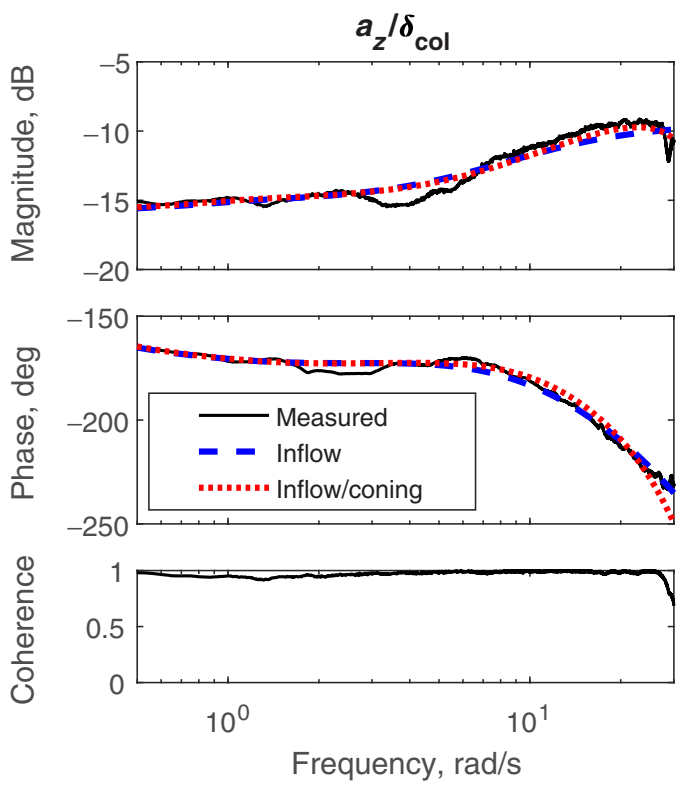

Fig. 5. Match in vertical acceleration for models with and without coning (hover).

\section{Regressive Lead-Lag}

The regressive flap responses to cyclic inputs are theoretically of second order but can be accurately modeled as two coupled first-order equations for system-identification purposes as shown in the section on flapping. No similar simplification exists for the coupled state-space equations for the lead-lag motion. Therefore, a modal approach is usually taken, where two closely spaced complex pole/zero pairs (dipoles) with a common denominator are appended to the pitch and roll rate responses to account for the effect of the regressive lead-lag (see chapter 15 in Ref. 12).

\section{Second-order dipole}

A complex (second-order) dipole with input $u$ and output $y$ and the transfer function (num $=$ numerator, den $=$ denominator)

$$
\frac{y}{u}=\frac{s^{2}+2 \zeta_{\mathrm{num}} \omega_{\mathrm{num}} s+\omega_{\mathrm{num}}^{2}}{s^{2}+2 \zeta_{\mathrm{den}} \omega_{\mathrm{den}} s+\omega_{\mathrm{den}}^{2}}
$$

can be modeled with the state equations

$$
\left(\begin{array}{l}
\dot{x}_{1} \\
\dot{x}_{2}
\end{array}\right)=\left[\begin{array}{cc}
0 & 1 \\
-\omega_{\mathrm{den}}^{2} & -2 \zeta_{\mathrm{den}} \omega_{\mathrm{den}}
\end{array}\right]\left(\begin{array}{l}
x_{1} \\
x_{2}
\end{array}\right)+\left[\begin{array}{l}
0 \\
1
\end{array}\right](u)
$$

and the output equation

$$
y=\left(\omega_{\text {num }}^{2}-\omega_{\text {den }}^{2}\right) x_{1}+\left(2 \zeta_{\text {num }} \omega_{\text {num }}-2 \zeta_{\text {den }} \omega_{\text {den }}\right) x_{2}+u
$$

Regarding the following transfer function of roll rate $(p)$ due to lateral cyclic input, where the first-order quasi-static response is augmented by a second-order lead-lag (11) dipole:

$$
\frac{p_{\mathrm{ll}}}{\delta_{\mathrm{lat}}}=\frac{L_{\delta_{\mathrm{lat}}}}{s-L_{p}} \frac{s^{2}+2 \zeta_{p} \omega_{p} s+\omega_{p}^{2}}{s^{2}+2 \zeta_{\mathrm{ll}} \omega_{\mathrm{ll}} s+\omega_{\mathrm{ll}}^{2}}
$$

it can be interpreted in two ways:

$$
\frac{p_{\text {ll }}}{\delta_{\text {lat }}}=\frac{p}{\delta_{\text {lat }}} \frac{p_{\text {ll }}}{p} \quad(\text { dipole at the output })
$$

or

$$
\left.\frac{p_{\mathrm{ll}}}{\delta_{\text {lat }}}=\frac{p_{\mathrm{ll}}}{\delta_{\text {lat,ll }}} \frac{\delta_{\text {lat,ll }}}{\delta_{\text {lat }}} \quad \text { (dipole at the input }\right)
$$

which leads to different state space implementations.

For the first case (dipole at the output), the equations for $p / \delta_{\text {lat }}$ and the dipole $p_{\mathrm{II}} / p$ (with $p$ as the input) are appended to each other in the state equations

$$
\left(\begin{array}{c}
\dot{p} \\
\dot{x}_{1} \\
\dot{x}_{2}
\end{array}\right)=\left[\begin{array}{ccc}
L_{p} & 0 & 0 \\
0 & 0 & 1 \\
1 & -\omega_{11}^{2} & -2 \zeta_{11} \omega_{\mathrm{ll}}
\end{array}\right]\left(\begin{array}{c}
p \\
x_{1} \\
x_{2}
\end{array}\right)+\left[\begin{array}{c}
L_{\delta_{\text {lat }}} \\
0 \\
0
\end{array}\right]\left(\delta_{\text {lat }}\right)
$$

and the roll rate including lead-lag $p_{\mathrm{ll}}$ is calculated by the output equation (see Eq. (23))

$$
p_{\mathrm{ll}}=p+\left(\omega_{p}^{2}-\omega_{1 \mathrm{ll}}^{2}\right) x_{1}+\left(2 \zeta_{p} \omega_{p}-2 \zeta_{\mathrm{ll}} \omega_{\mathrm{ll}}\right) x_{2}
$$

For the second case (dipole at the input), the input $\delta_{\text {lat }}$ has to be replaced by $\delta_{\text {lat,ll }}$ in the equation for $\dot{p}_{\text {ll }}$. This leads to the following system:

$$
\left(\begin{array}{c}
\dot{p}_{\mathrm{ll}} \\
\dot{x}_{1} \\
\dot{x}_{2}
\end{array}\right)=\left[\begin{array}{ccc}
L_{p} & L_{x_{1}} & L_{x_{2}} \\
0 & 0 & 1 \\
0 & -\omega_{\mathrm{ll}}^{2} & -2 \zeta_{\mathrm{ll}} \omega_{\mathrm{ll}}
\end{array}\right]\left(\begin{array}{c}
p_{\mathrm{ll}} \\
x_{1} \\
x_{2}
\end{array}\right)+\left[\begin{array}{c}
L_{\delta_{\mathrm{lat}}} \\
0 \\
1
\end{array}\right]\left(\delta_{\mathrm{lat}}\right)
$$

with

$$
L_{x_{1}}=L_{\delta_{\text {lat }}}\left(\omega_{p}^{2}-\omega_{11}^{2}\right) \quad L_{x_{2}}=L_{\delta_{\text {lat }}}\left(2 \zeta_{p} \omega_{p}-2 \zeta_{11} \omega_{11}\right)
$$

No output equation is required in this case because the transformation from $\delta_{\text {lat }}$ to $\delta_{\text {lat,Il }}$ is already contained in the state equations.

\section{Extension to two inputs and two outputs}

If a second-order dipole with the same denominator is also appended to the pitch axis and if both inputs $\delta_{\text {lon }}$ and $\delta_{\text {lat }}$ act on $p$ and $q$, the first formulation with the dipoles at the output leads to state equations

$$
\begin{aligned}
\left(\begin{array}{c}
\dot{p} \\
\dot{q} \\
\dot{x}_{1} \\
\dot{x}_{2} \\
\dot{y}_{1} \\
\dot{y}_{2}
\end{array}\right)= & {\left[\begin{array}{cccccc}
L_{p} & 0 & 0 & 0 & 0 & 0 \\
0 & M_{q} & 0 & 0 & 0 & 0 \\
0 & 0 & 0 & 1 & 0 & 0 \\
1 & 0 & -\omega_{\mathrm{ll}}^{2}-2 \zeta_{\mathrm{ll}} \omega_{\mathrm{ll}} & 0 & 0 \\
0 & 0 & 0 & 0 & 0 & 1 \\
0 & 1 & 0 & 0 & -\omega_{\mathrm{ll}}^{2} & -2 \zeta_{\mathrm{ll}} \omega_{\mathrm{ll}}
\end{array}\right]\left(\begin{array}{c}
p \\
q \\
x_{1} \\
x_{2} \\
y_{1} \\
y_{2}
\end{array}\right) } \\
& +\left[\begin{array}{ccc}
L_{\delta_{\text {lon }}} & L_{\delta_{\text {lat }}} \\
M_{\delta_{\text {lon }}} & M_{\delta_{\text {lat }}} \\
0 & 0 \\
0 & 0 \\
0 & 0 \\
0 & 0
\end{array}\right]\left(\begin{array}{c}
\delta_{\text {lon }} \\
\delta_{\text {lat }}
\end{array}\right)
\end{aligned}
$$

and output equations

$$
\begin{aligned}
& p_{\mathrm{ll}}=p+\left(\omega_{p}^{2}-\omega_{\mathrm{ll}}^{2}\right) x_{1}+\left(2 \zeta_{p} \omega_{p}-2 \zeta_{\mathrm{ll}} \omega_{\mathrm{ll}}\right) x_{2} \\
& q_{\mathrm{ll}}=q+\left(\omega_{q}^{2}-\omega_{\mathrm{ll}}^{2}\right) x_{1}+\left(2 \zeta_{q} \omega_{q}-2 \zeta_{\mathrm{ll}} \omega_{\mathrm{ll}}\right) x_{2}
\end{aligned}
$$

The resulting transfer functions are

$$
\begin{array}{ll}
\frac{p_{\mathrm{ll}}}{\delta_{\text {lon }}}=\frac{L_{\delta_{\text {lon }}}}{s-L_{p}} \frac{\left[\zeta_{p}, \omega_{p}\right]}{\left[\zeta_{\mathrm{ll}}, \omega_{\mathrm{ll}}\right]} & \frac{p_{\mathrm{ll}}}{\delta_{\mathrm{lat}}}=\frac{L_{\delta_{\mathrm{lat}}}}{s-L_{p}} \frac{\left[\zeta_{p}, \omega_{p}\right]}{\left[\zeta_{\mathrm{ll}}, \omega_{\mathrm{ll}}\right]} \\
\frac{q_{\mathrm{ll}}}{\delta_{\text {lon }}}=\frac{M_{\delta_{\mathrm{lon}}}}{s-M_{q}} \frac{\left[\zeta_{q}, \omega_{q}\right]}{\left[\zeta_{\mathrm{ll}}, \omega_{\mathrm{ll}}\right]} & \frac{q_{\mathrm{ll}}}{\delta_{\mathrm{lat}}}=\frac{M_{\delta_{\mathrm{lat}}}}{s-M_{q}} \frac{\left[\zeta_{q}, \omega_{q}\right]}{\left[\zeta_{\mathrm{ll}}, \omega_{\mathrm{ll}}\right]}
\end{array}
$$

This means that the dipoles in $p_{\mathrm{ll}} / \delta_{\text {lon }}$ and $p_{\mathrm{ll}} / \delta_{\text {lat }}$ are identical. The same holds for the dipoles in $q_{\mathrm{ll}} / \delta_{\text {lon }}$ and $q_{\mathrm{ll}} / \delta_{\text {lat }}$. 

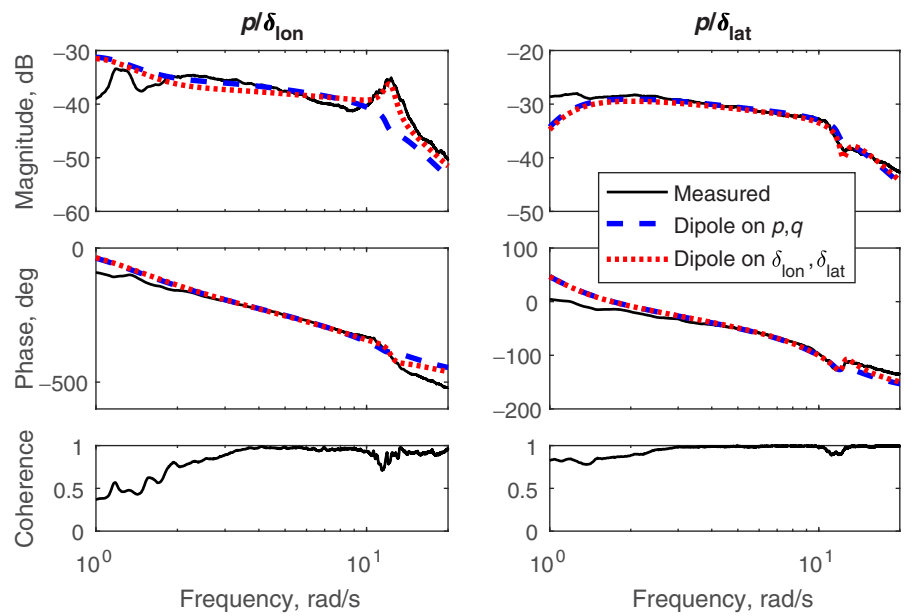

Fig. 6. Match in roll rate due to cyclic inputs for both lead-lag models (hover).

For the ACT/FHS the modeling variant with the dipoles at the input was used, where two second-order dipoles act on the longitudinal and lateral cyclic inputs as described in Ref. 19. The corresponding state equations are

$$
\begin{aligned}
\left(\begin{array}{l}
\dot{p}_{\mathrm{ll}} \\
\dot{q}_{\mathrm{ll}} \\
\dot{x}_{1} \\
\dot{x}_{2} \\
\dot{y}_{1} \\
\dot{y}_{2}
\end{array}\right)= & {\left[\begin{array}{cccccc}
L_{p} & 0 & L_{x_{1}} & L_{x_{2}} & L_{y_{1}} & L_{y_{2}} \\
0 & M_{q} & M_{x_{1}} & M_{x_{2}} & M_{y_{1}} & M_{y_{2}} \\
0 & 0 & 0 & 1 & 0 & 0 \\
0 & 0 & -\omega_{\mathrm{ll}}^{2} & -2 \zeta_{\mathrm{ll}} \omega_{\mathrm{ll}} & 0 & 0 \\
0 & 0 & 0 & 0 & 0 & 1 \\
0 & 0 & 0 & 0 & -\omega_{\mathrm{ll}}^{2} & -2 \zeta_{\mathrm{ll}} \omega_{\mathrm{ll}}
\end{array}\right]\left(\begin{array}{c}
p_{\mathrm{ll}} \\
q_{\mathrm{ll}} \\
x_{1} \\
x_{2} \\
y_{1} \\
y_{2}
\end{array}\right) } \\
& +\left[\begin{array}{cc}
L_{\delta_{\text {lon }}} & L_{\delta_{\text {lat }}} \\
M_{\delta_{\text {lon }}} & M_{\delta_{\text {lat }}} \\
0 & 0 \\
1 & 0 \\
0 & 0 \\
0 & 1
\end{array}\right]\left(\begin{array}{c}
\delta_{\text {lon }} \\
\delta_{\text {lat }}
\end{array}\right)
\end{aligned}
$$

with

$$
\begin{aligned}
& L_{x_{1}}=L_{\delta_{\text {lon }}}\left(\omega_{x p}^{2}-\omega_{\text {ll }}^{2}\right) \quad L_{x_{2}}=L_{\delta_{\text {lon }}}\left(2 \zeta_{x p} \omega_{x p}-2 \zeta_{11} \omega_{\text {ll }}\right) \\
& L_{y_{1}}=L_{\delta_{\text {lat }}}\left(\omega_{y p}^{2}-\omega_{\text {ll }}^{2}\right) \quad L_{y_{2}}=L_{\delta_{\text {lat }}}\left(2 \zeta_{y p} \omega_{y p}-2 \zeta_{11} \omega_{\mathrm{ll}}\right) \\
& M_{x_{1}}=M_{\delta_{\text {lon }}}\left(\omega_{x q}^{2}-\omega_{\text {ll }}^{2}\right) \quad M_{x_{2}}=M_{\delta_{\text {lon }}}\left(2 \zeta_{x q} \omega_{x q}-2 \zeta_{11} \omega_{11}\right) \\
& M_{y_{1}}=M_{\delta_{\text {lat }}}\left(\omega_{y q}^{2}-\omega_{11}^{2}\right) \quad M_{y_{2}}=M_{\delta_{\text {lat }}}\left(2 \zeta_{y q} \omega_{y q}-2 \zeta_{11} \omega_{11}\right)
\end{aligned}
$$

The resulting transfer functions are

$$
\begin{array}{cc}
\frac{p_{\mathrm{ll}}}{\delta_{\mathrm{lon}}}=\frac{L_{\delta_{\mathrm{lon}}}}{s-L_{p}} \frac{\left[\zeta_{x p}, \omega_{x p}\right]}{\left[\zeta_{\mathrm{ll}}, \omega_{\mathrm{ll}}\right]} \quad \frac{p_{\mathrm{ll}}}{\delta_{\mathrm{lat}}}=\frac{L_{\delta_{\mathrm{lat}}}}{s-L_{p}} \frac{\left[\zeta_{y p}, \omega_{y p}\right]}{\left[\zeta_{\mathrm{ll}}, \omega_{\mathrm{ll}}\right]} \\
\frac{q_{\mathrm{ll}}}{\delta_{\mathrm{lon}}}=\frac{M_{\delta_{\mathrm{lon}}}}{s-M_{q}} \frac{\left[\zeta_{x q}, \omega_{x q}\right]}{\left[\zeta_{\mathrm{ll}}, \omega_{\mathrm{ll}}\right]} & \frac{q_{\mathrm{ll}}}{\delta_{\mathrm{lat}}}=\frac{M_{\delta_{\mathrm{lat}}}}{s-M_{q}} \frac{\left[\zeta_{y q}, \omega_{y q}\right]}{\left[\zeta_{11}, \omega_{\mathrm{ll}}\right]}
\end{array}
$$

and thus the dipoles in $p_{\mathrm{ll}} / \delta_{\mathrm{lon}}$ and $p_{\mathrm{ll}} / \delta_{\mathrm{lat}}$ have different numerators and are therefore not identical. The same holds for the dipoles in $q_{11} / \delta_{\text {lon }}$ and $q_{\mathrm{ll}} / \delta_{\text {lat }}$.

Figure 6 shows that the lead-lag dipoles for the ACT/FHS in $p / \delta_{\text {lon }}$ and $p / \delta_{\text {lat }}$ are different and can thus only be captured by the model with two dipoles at the inputs and not by the usual formulation with dipoles at the outputs.

Another advantage of the model formulation with the dipoles at the inputs is that influences of the lead-lag on the longitudinal and
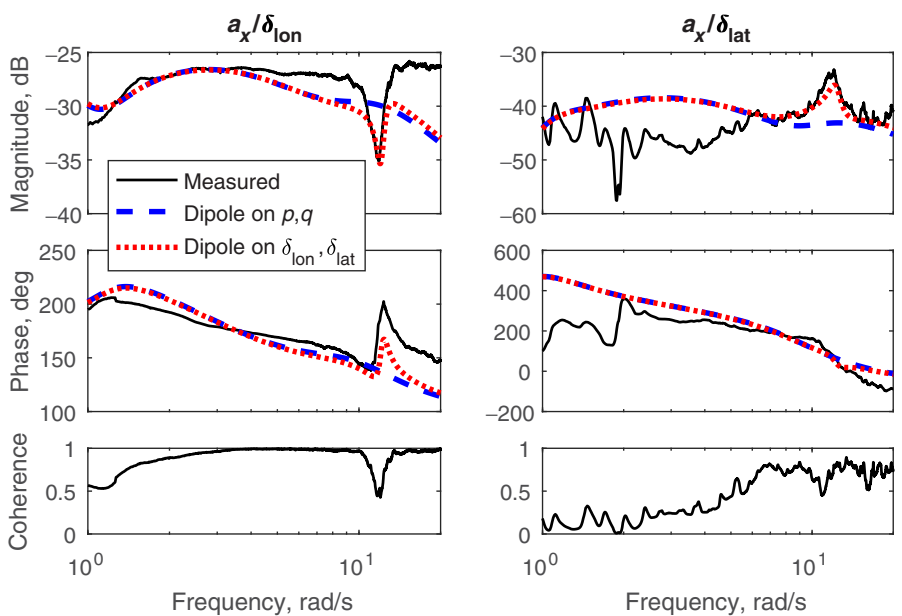

Fig. 7. Match in longitudinal acceleration due to cyclic inputs for both lead-lag models (hover).
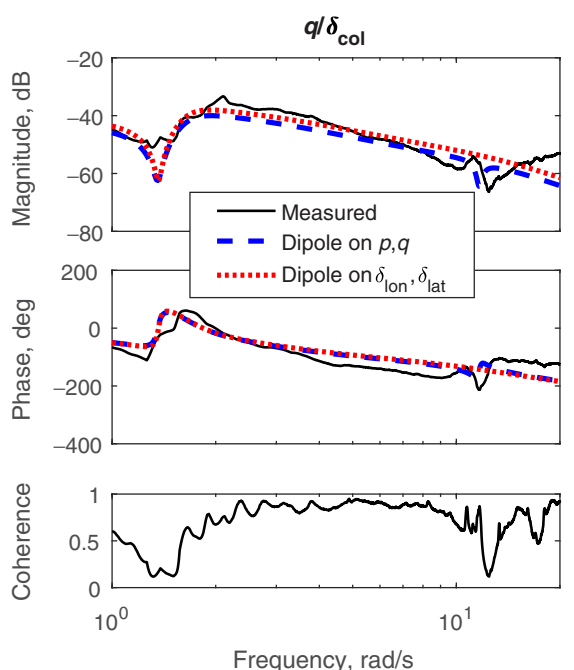

Fig. 8. Match in pitch rate due to collective input for both lead-lag models (30 kt).

lateral accelerations that are present in ACT/FHS data (see Fig. 7) can be captured.

On the other hand, the modeling variant with the dipoles on the outputs has the advantage, that the lead-lag influence can also be modeled for collective inputs (see, e.g., Fig. 8). For the model with input dipoles, this would require a third dipole and thus two additional state variables.

\section{Formulation with one second-order dipole}

At DLR, system identification for the ACT/FHS has also been performed using the PBSIDopt method (see Ref. 15). This method does not require specifying a model structure beforehand, but instead the model states are determined along with the corresponding model parameters. The physical interpretation of the resulting models, however, is difficult because the model states cannot be specified, and the system matrices are fully populated. Nevertheless, from comparing the eigenvalues of the identified models, it was obvious that the PBSIDopt-identified models needed only one dipole to model the regressive lead-lag.

Thus, a new model formulation for the regressive lead-lag was developed where one dipole (with states $z_{1}$ and $z_{2}$ ) is triggered by both the 
longitudinal and lateral cyclic inputs:

$$
\begin{aligned}
\left(\begin{array}{c}
\dot{p}_{\mathrm{ll}} \\
\dot{q}_{\mathrm{ll}} \\
\dot{z}_{1} \\
\dot{z}_{2}
\end{array}\right)= & {\left[\begin{array}{cccc}
L_{p} & 0 & L_{z_{1}} & L_{z_{2}} \\
0 & M_{q} & M_{z_{1}} & M_{z_{2}} \\
0 & 0 & 0 & 1 \\
0 & 0 & -\omega_{\text {ll }}^{2} & -2 \zeta_{\mathrm{ll}} \omega_{\mathrm{ll}}
\end{array}\right]\left(\begin{array}{c}
p_{\mathrm{ll}} \\
q_{\mathrm{ll}} \\
z_{1} \\
z_{2}
\end{array}\right) } \\
& +\left[\begin{array}{cc}
L_{\delta_{\text {lon }}} & L_{\delta_{\text {lat }}} \\
M_{\delta_{\text {lon }}} & M_{\delta_{\text {lat }}} \\
D_{\delta_{\text {lon }}} & D_{\delta_{\text {lat }}} \\
1 & 1
\end{array}\right]\left(\begin{array}{c}
\delta_{\text {lon }} \\
\delta_{\text {lat }}
\end{array}\right)
\end{aligned}
$$

This results in the following transfer functions:

$$
\begin{aligned}
\frac{p_{\mathrm{ll}}}{\delta_{\mathrm{lon}}} & =\frac{1}{s-L_{p}} \frac{L_{\delta_{\mathrm{lon}}} s^{2}+P_{x_{1}} s+P_{x_{0}}}{\left[\zeta_{\mathrm{ll}}, \omega_{\mathrm{ll}}\right]} \\
\frac{p_{\mathrm{ll}}}{\delta_{\mathrm{lat}}} & =\frac{1}{s-L_{p}} \frac{L_{\delta_{\mathrm{lat}}} s^{2}+P_{y_{1}} s+P_{y_{0}}}{\left[\zeta_{\mathrm{ll}}, \omega_{\mathrm{ll}}\right]} \\
\frac{q_{\mathrm{ll}}}{\delta_{\mathrm{lon}}} & =\frac{1}{s-M_{q}} \frac{M_{\delta_{\mathrm{lon}}} s^{2}+Q_{x_{1}} s+Q_{x_{0}}}{\left[\zeta_{\mathrm{ll}}, \omega_{\mathrm{ll}}\right]} \\
\frac{q_{\mathrm{ll}}}{\delta_{\mathrm{lat}}} & =\frac{1}{s-M_{q}} \frac{M_{\delta_{\mathrm{lat}}} s^{2}+Q_{y_{1}} s+Q_{y_{0}}}{\left[\zeta_{\mathrm{ll}}, \omega_{\mathrm{ll}}\right]}
\end{aligned}
$$

with

$$
\begin{aligned}
& P_{x_{1}}=L_{z_{2}}+L_{z_{1}} D_{\delta_{\mathrm{lon}}}+L_{\delta_{\mathrm{lon}}} 2 \zeta_{\mathrm{ll}} \omega_{\mathrm{ll}} \\
& P_{x_{0}}=L_{z_{1}}+L_{\delta_{\mathrm{lon}}} \omega_{\mathrm{ll}}^{2}-L_{z_{2}} D_{\delta_{\mathrm{lon}}} \omega_{\mathrm{ll}}^{2}+L_{z_{1}} D_{\delta_{\mathrm{lon}}} 2 \zeta_{\mathrm{ll}} \omega_{\mathrm{ll}} \\
& P_{y_{1}}=L_{z_{2}}+L_{z_{1}} D_{\delta_{\mathrm{lat}}}+L_{\delta_{\mathrm{lat}}} 2 \zeta_{\mathrm{ll}} \omega_{\mathrm{ll}} \\
& P_{y_{0}}=L_{z_{1}}+L_{\delta_{\mathrm{lat}}} \omega_{\mathrm{ll}}^{2}-L_{z_{2}} D_{\delta_{\mathrm{lat}}} \omega_{\mathrm{ll}}^{2}+L_{z_{1}} D_{\delta_{\mathrm{lat}}} 2 \zeta_{\mathrm{ll}} \omega_{\mathrm{ll}} \\
& Q_{x_{1}}=M_{z_{2}}+M_{z_{1}} D_{\delta_{\mathrm{lon}}}+M_{\delta_{\mathrm{lon}}} 2 \zeta_{\mathrm{ll}} \omega_{\mathrm{ll}} \\
& Q_{x_{0}}=M_{z_{1}}+M_{\delta_{\mathrm{lon}}} \omega_{\mathrm{ll}}^{2}-M_{z_{2}} D_{\delta_{\mathrm{lon}}} \omega_{1 \mathrm{ll}}^{2}+M_{z_{1}} D_{\delta_{\mathrm{lon}}} 2 \zeta_{\mathrm{ll}} \omega_{\mathrm{ll}} \\
& Q_{y_{1}}=M_{z_{2}}+M_{z_{1}} D_{\delta_{\mathrm{lat}}}+M_{\delta_{\mathrm{lat}}} 2 \zeta_{\mathrm{ll}} \omega_{\mathrm{ll}} \\
& Q_{y_{0}}=M_{z_{1}}+M_{\delta_{\mathrm{lat}}} \omega_{\mathrm{ll}}^{2}-M_{z_{2}} D_{\delta_{\mathrm{lat}}} \omega_{1 \mathrm{ll}}^{2}+M_{z_{1}} D_{\delta_{\mathrm{lat}}} 2 \zeta_{\mathrm{ll}} \omega_{\mathrm{ll}}
\end{aligned}
$$

Like for the formulation with two dipoles at the inputs, all dipole numerators are different. This means that different dipoles in $p_{\mathrm{ll}} / \delta_{\text {lon }}$ and $p_{\mathrm{ll}} / \delta_{\text {lat }}$, respectively, $q_{\mathrm{ll}} / \delta_{\text {lon }}$ and $q_{\mathrm{ll}} / \delta_{\text {lat }}$ can be realized also with the one-dipole formulation. In the appendix, it is shown analytically that the parameters of the two-dipole model can be calculated from those of the one-dipole formulation when the pitch and roll equations are decoupled.

Figure 9 shows that this one-dipole approach leads to results that are comparable to those obtained with two dipoles at the input.

Furthermore, this one-dipole approach can easily be extended so that the lead-lag is also excited by collective inputs by just adding another column in the control matrix from Eq. (37). Thus the one-dipole formulation has the advantages of both of the other two-dipole modeling variants and is simpler because it needs only two lead-lag states.

\section{Engine Modeling}

After accounting for the rotor DOFs of flapping, inflow, and regressive lead-lag, the identified models of the ACT/FHS still had deficits that were attributed to missing engine dynamics. According to Ref. 12, the effect of the engine/governor dynamics on the fuselage response primarily manifests itself as a large additional phase lag in the $p / \delta_{\mathrm{col}}, q / \delta_{\mathrm{col}}$, and
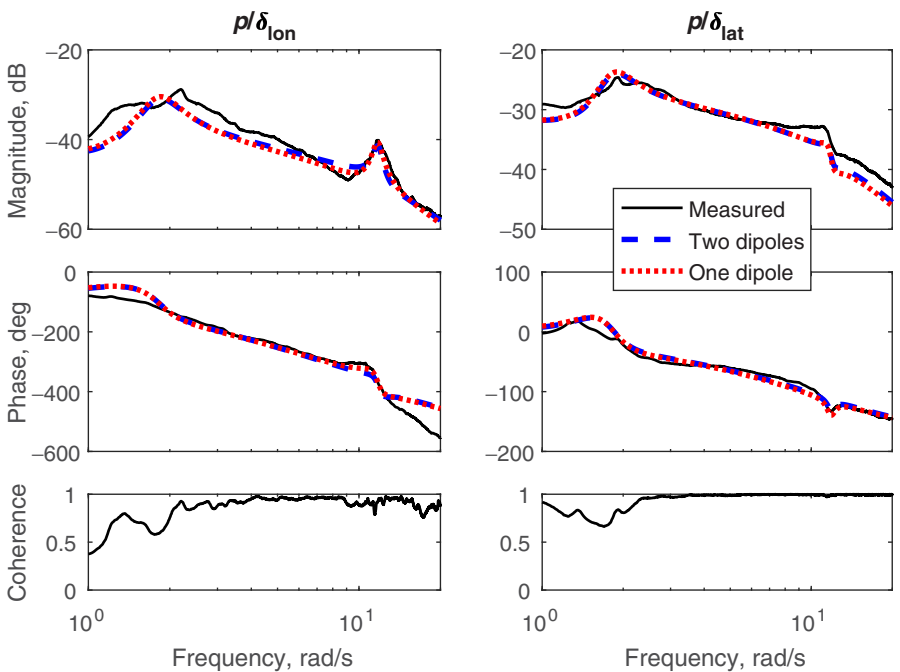

Fig. 9. Match in roll rate due to cyclic inputs for the one- and twodipole formulations $(60 \mathrm{kt})$.

$r / \delta_{\text {col }}$ frequency responses. In case of the ACT/FHS, deficits were only experienced in the response of yaw rate due to collective input.

\section{Preliminary investigations}

First, it was investigated whether accounting for engine dynamics by a simple lag in the influence of collective input on the angular rates as suggested in chapter 15 of Ref. 12 would be sufficient. For this approach, the time delay in the collective input was approximated by a Padé approximation:

$$
\frac{\delta_{\mathrm{col}}^{\prime}}{\delta_{\mathrm{col}}}=\frac{2 / \tau_{\mathrm{en}}-s}{2 / \tau_{\mathrm{en}}+s}
$$

where $\tau_{\mathrm{en}}$ is the time constant. $\delta_{\mathrm{col}}$ is the original collective input, and $\delta_{\mathrm{col}}^{\prime}$ is the delayed collective input, that is used in the equations of motion for the pitch, roll, and yaw rates. This approach, however, did not yield the desired improvement.

Therefore, a linear regression was performed in the time domain using the equation for yaw acceleration

$$
\dot{r}=N_{r} r+N_{v} v+N_{p} p+N_{\delta_{\mathrm{col}}} \delta_{\mathrm{col}}+N_{\delta_{\text {ped }}} \delta,+N_{Q} Q+N_{\Omega} \Omega
$$

The coefficients $N_{r}, \ldots N_{\Omega}$ were determined using measured time history data both for the output variable $\dot{r}$ and the inputs $r, \ldots, \Omega$. This investigation showed that torque $Q$ has a profound influence on the yawing motion whereas no direct influence of rotor speed $\Omega$ could be found. Thus, torque modeling with a dynamic engine model was needed.

\section{Frequency response modeling}

First, the frequency response for rotor speed due to collective was approximated separately. A good approximation was reached with the following model:

$$
\frac{\Omega}{\delta_{\mathrm{col}}}=\frac{K_{\delta_{\mathrm{col}}}}{s^{2}+2 \zeta_{\mathrm{en}} \omega_{\mathrm{en}} s+\omega_{\mathrm{en}}^{2}} \frac{s-E_{z, \mathrm{col}}}{s-E_{p}}
$$

which consists of a second-order system with frequency $\omega_{\text {en }}$ and damping $\zeta_{\text {en }}$ combined by a first-order pole/zero pair (dipole) with pole $E_{p}$ and zero $E_{z, \mathrm{col}}$. 

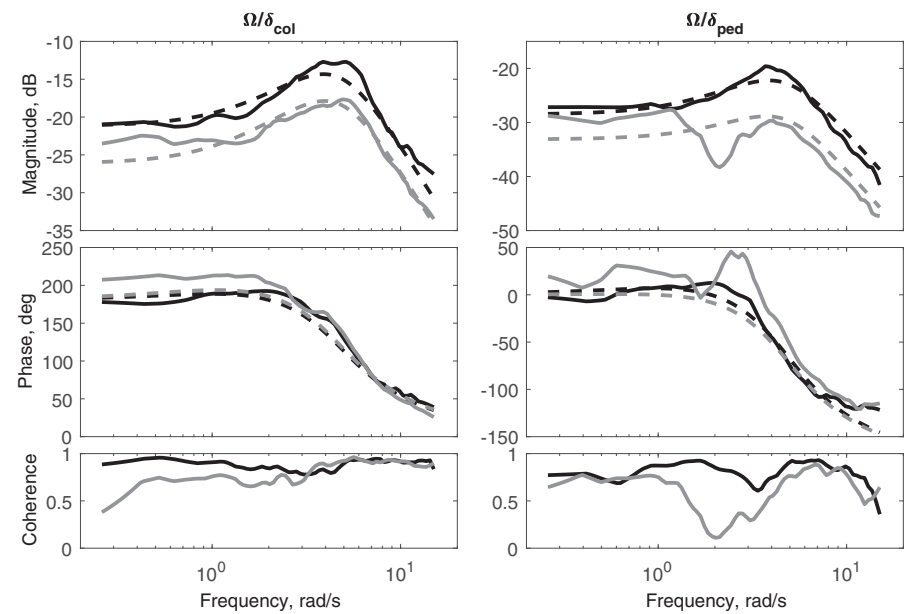

Fig. 10. Transfer function approximation for rotor speed $($ black $=$ hover, gray $=90 \mathrm{kt}$, solid $=$ measured, dashed $=$ model $)$.

In the engine model that was identified for the Firescout UAV (see Ref. 20), a second-order system for rotor speed is combined with a washout filter modeling the governor dynamics. This washout filter corresponds to setting $E_{z, \mathrm{col}}=0$ in Eq. (42). The engine of the ACT/FHS is controlled by a FADEC (full authority digital engine control) system, and a sufficient approximation of $\Omega / \delta_{\text {col }}$ could be reached only with $E_{z, \mathrm{col}} \neq 0$.

Next, the frequency responses due to collective and pedal inputs were approximated with a common denominator. This corresponds to extending Eq. (42) by

$$
\frac{\Omega}{\delta_{\text {ped }}}=\frac{K_{\delta_{\text {ped }}}}{s^{2}+2 \zeta_{\text {en }} \omega_{\text {en }} s+\omega_{\text {en }}^{2}} \frac{s-E_{z, \text { ped }}}{s-E_{p}}
$$

Figure 10 shows the resulting match for two of the five reference speeds. (The drop in coherence around $2 \mathrm{rad} / \mathrm{s}$ in $\Omega / \delta_{\text {ped }}$ for the $90 \mathrm{kt}$ case is caused by the influence of dutch roll dynamics.)

\section{State-space model}

For inclusion in the identification model, the frequency response models from Eqs. (42) and (43) had to be converted into a state-space model. For this, a common zero $E_{z \text {,col }}=E_{z \text {,ped }}=E_{z}$ was enforced. Defining an instrumental variable $\Omega_{\mathrm{en}}$ with

$$
\frac{\Omega}{\delta_{\mathrm{col}}}=\frac{\Omega}{\Omega_{\mathrm{en}}} \frac{\Omega_{\mathrm{en}}}{\delta_{\mathrm{col}}}=\frac{s-E_{z}}{s-E_{p}} \frac{K_{\delta_{\mathrm{col}}}}{s^{2}+2 \zeta_{\mathrm{en}} \omega_{\mathrm{en}} s+\omega_{\mathrm{en}}^{2}}
$$

yields the two equations

$$
\begin{aligned}
\ddot{\Omega}_{\mathrm{en}} & =-2 \zeta_{\mathrm{en}} \omega_{\mathrm{en}} \dot{\Omega}_{\mathrm{en}}-\omega_{\mathrm{en}}^{2} \Omega_{\mathrm{en}}+K_{\delta_{\mathrm{col}}} \delta_{\mathrm{col}} \\
\dot{\Omega} & =\dot{\Omega}_{\mathrm{en}}-E_{z} \Omega_{\mathrm{en}}+E_{p} \Omega
\end{aligned}
$$

Adding the pedal input and using matrix notation yields

$$
\begin{aligned}
\left(\begin{array}{c}
\ddot{\Omega}_{\mathrm{en}} \\
\dot{\Omega}_{\mathrm{en}} \\
\dot{\Omega}
\end{array}\right)= & {\left[\begin{array}{ccc}
-2 \zeta_{\mathrm{en}} \omega_{\mathrm{en}} & -\omega_{\mathrm{en}}^{2} & 0 \\
1 & 0 & 0 \\
1 & -E_{z} & E_{p}
\end{array}\right]\left(\begin{array}{c}
\dot{\Omega}_{\mathrm{en}} \\
\Omega_{\mathrm{en}} \\
\Omega
\end{array}\right) } \\
& +\left[\begin{array}{cc}
K_{\delta_{\mathrm{col}}} & K_{\delta_{\mathrm{ped}}} \\
0 & 0 \\
0 & 0
\end{array}\right]\left(\begin{array}{c}
\delta_{\mathrm{col}} \\
\delta_{\text {ped }}
\end{array}\right)
\end{aligned}
$$

Tischler (chapter 12 in Ref. 12) suggests using a Taylor series for modeling the torque dynamics:

$$
\dot{Q}=R_{Q} Q+R_{\Omega} \Omega+R_{\dot{\Omega}} \dot{\Omega}+R_{\delta_{\mathrm{col}}} \delta_{\mathrm{col}}+R_{\delta_{\mathrm{ped}}} \delta_{\text {ped }}
$$

Inserting the equations for $\dot{\Omega}$ from Eq. (45) yields

$$
\begin{aligned}
\dot{Q}= & R_{\dot{\Omega}} \dot{\Omega}_{\mathrm{en}}-E_{z} R_{\dot{\Omega}} \Omega_{\mathrm{en}}+\left(R_{\Omega}+E_{p} R_{\dot{\Omega}}\right) \Omega \\
& +R_{Q} Q+R_{\delta_{\mathrm{col}}} \delta_{\mathrm{col}}+R_{\delta_{\mathrm{ped}}} \delta_{\mathrm{ped}}
\end{aligned}
$$

This equations was added to the system from Eq. (46) to arrive at the desired state space system for rotor speed and torque:

$$
\begin{aligned}
\left(\begin{array}{c}
\ddot{\Omega}_{\mathrm{en}} \\
\dot{\Omega}_{\mathrm{en}} \\
\dot{\Omega} \\
\dot{Q}
\end{array}\right)= & {\left[\begin{array}{cccc}
-2 \zeta_{\mathrm{en}} \omega_{\mathrm{en}} & -\omega_{\mathrm{en}}^{2} & 0 & 0 \\
1 & 0 & 0 & 0 \\
1 & -E_{z} & E_{p} & 0 \\
R_{\dot{\Omega}} & -E_{z} R_{\dot{\Omega}} & R_{\Omega}+E_{p} R_{\dot{\Omega}} & R_{Q}
\end{array}\right]\left(\begin{array}{c}
\dot{\Omega}_{\mathrm{en}} \\
\Omega_{\mathrm{en}} \\
\Omega \\
Q
\end{array}\right) } \\
& +\left[\begin{array}{cc}
K_{\delta_{\mathrm{col}}} & K_{\delta_{\mathrm{ped}}} \\
0 & 0 \\
0 & 0 \\
R_{\delta_{\mathrm{col}}} & R_{\delta_{\mathrm{ped}}}
\end{array}\right]\left(\begin{array}{c}
\delta_{\mathrm{col}} \\
\delta_{\mathrm{ped}}
\end{array}\right)
\end{aligned}
$$

The parameters of this model were identified for each of the five reference speeds with the maximum likelihood (ML) frequency domain method using collective and pedal sweep maneuvers. The parameter $R_{\dot{\Omega}}$ exhibited high uncertainty and was thus set to zero. The identified models were validated with 3211 multistep maneuvers. Figure 11 shows that the match in torque and rotor speed both for collective and pedal inputs in hover is quite good.

\section{Model simplifications}

Before the development of the models described in this paper, corrections in transfer function form had been used to reduce the remaining model deficits of the prior models as described in Ref. 21. As the corrections to $r / \delta_{\text {col }}$ that were needed to account for the missing engine dynamics used the same transfer function for all speeds, simplification of the identified engine model was attempted accordingly.

First the calculation of torque from rotor speed was investigated by using the measured data for $\Omega$ and $\dot{\Omega}$ in Eq. (47). This showed that the relationship between rotor speed and torque does not vary with speed. Also, the pedal influence coefficient $R_{\delta_{\text {ped }}}$ could be neglected.

Next, simplification of the rotor speed model was attempted. As the frequency responses from collective and pedal to $\Omega$ have a similar shape for all speeds and mainly a different amplitude (see Fig. 10), a model for rotor speed was determined where the parameters $\zeta_{\mathrm{en}}, \omega_{\mathrm{en}}, E_{z}$, and $E_{p}$ are identical for all speeds and only the control derivatives $K_{\delta_{\mathrm{col}}}$ and $K_{\delta_{\text {ped }}}$ are different (see Eq. (46)). The match of this simplified model was almost as good as for the models that had been optimized separately for each reference speed.

\section{Model integration}

Finally, the identified engine model was coupled to the overall model. Figure 12 shows the match in the frequency responses $r / \delta_{\text {ped }}$ and $r / \delta_{\text {col }}$ in hover for the models with and without engine modeling. As expected, the yaw rate response to collective input improves both in amplitude and in phase.

As can be seen from Fig. 12, the measured yaw rate response to pedal inputs (on-axis response) exhibits a first-order response (amplitude drop of $20 \mathrm{~dB}$ per decade) that is superimposed by some additional dynamics. These superimposed dynamics could be caused by the fenestron 


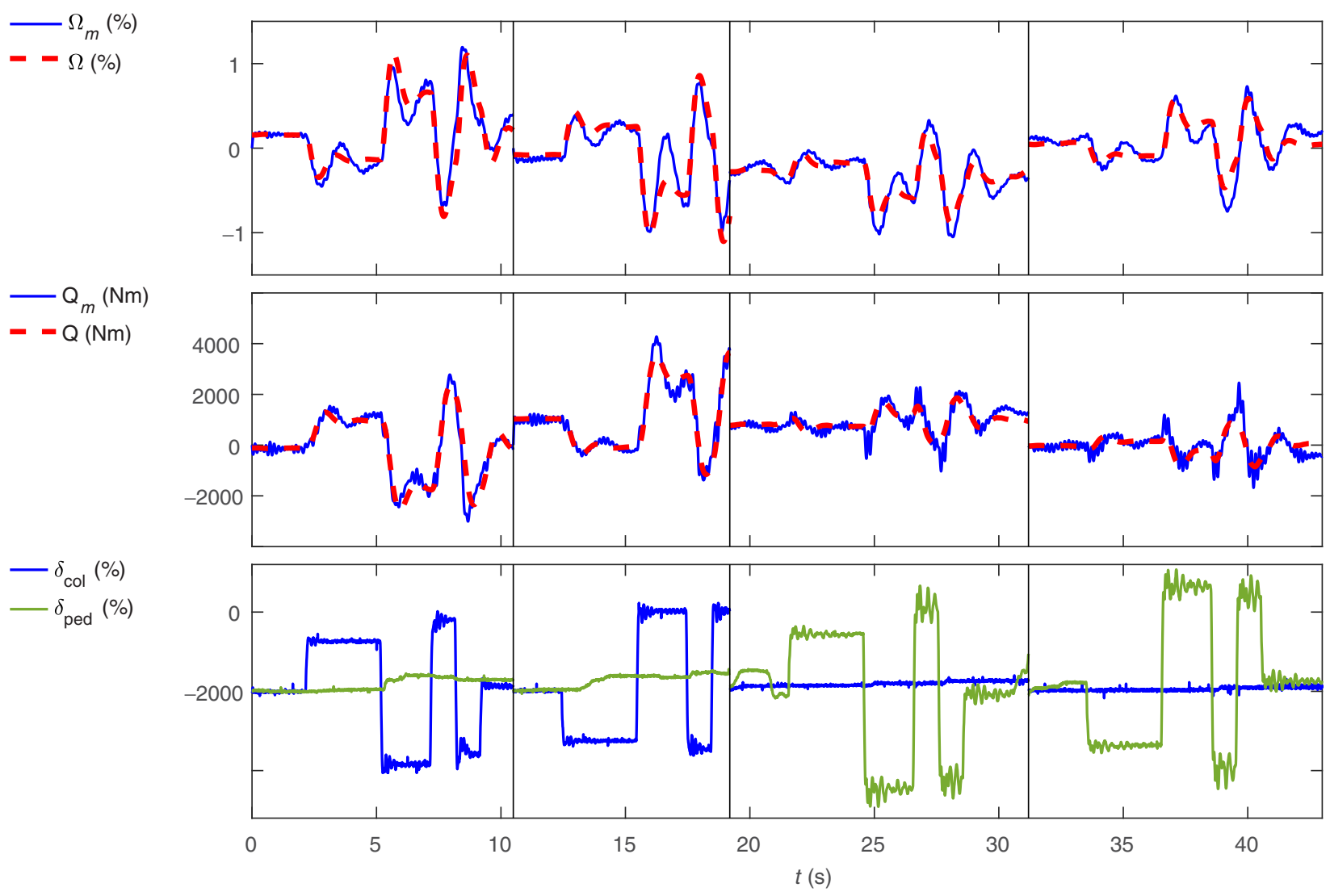

Fig. 11. Validation of the identified engine model in hover.
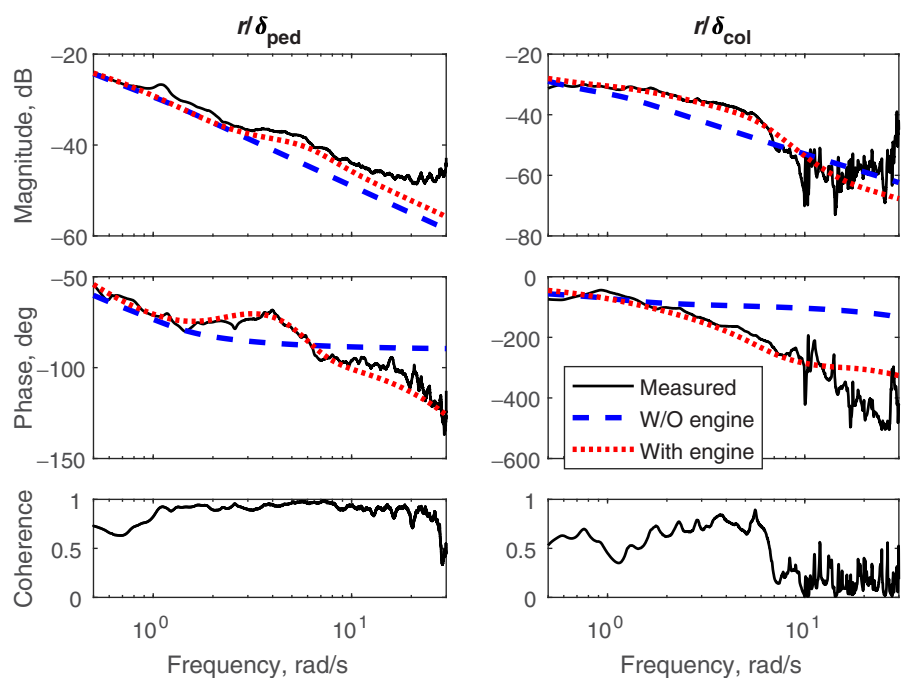

Fig. 12. Improvement of yaw responses by engine modeling (hover).

(see Ref. 22) or by engine dynamics and are not covered by the pure rigid-body modeling of this axis from the model without engine dynamics. Including the engine model clearly improves the match of this on-axis response so that the superimposed dynamics in $r / \delta_{\text {ped }}$ are probably caused by the FADEC system.

Thus accounting for engine dynamics improves the yaw response to both pedal and collective inputs, especially in hover.

\section{Integrated Model}

The overall models including rotor and engine dynamics were identified in an incremental way. The ML frequency domain method was used for all state space model identification.

First, six-DOF models were identified over the frequency range of $0.1-10 \mathrm{rad} / \mathrm{s}$. Next, the flapping dynamics were added using the modified explicit formulation as described in this paper. The theoretical prediction from Eq. (7) was used as a starting value for the flapping time constant, and the frequency range for the approximation was extended to 0.5$15 \mathrm{rad} / \mathrm{s}$. To extend the model range of validity even further, the explicit inflow equations were then added to the model and all model parameters optimized for a frequency range of $0.5-30 \mathrm{rad} / \mathrm{s}$.

To allow for model stitching, care was taken in every step, that it would be possible to interpolate between the models identified for each of the five reference speeds. Figure 13 shows the identified moment derivatives versus speed. It can be seen that the models for the different reference speeds have similar sets of free parameters and that the change over speed for all derivatives is smooth. Spline interpolation is used to interpolate between the different reference speeds.

Next, the engine dynamics were added. For this step, the engine model parameters were fixed at the identified values from the separate engine model identification. Coupling derivatives $N_{Q}$ and $L_{Q}$ (the latter only for hover and low speed) were introduced and estimated together with fuselage and rotor derivatives over a frequency range of $0.5-30 \mathrm{rad} / \mathrm{s}$.

For the ACT/FHS, the influence of the regressive lead-lag mode is only a very local effect around $12 \mathrm{rad} / \mathrm{s}$ (see Figs. 6 and 7) and thus could be identified last. The one-dipole formulation was used, and extending it to collective inputs was not necessary because the lead-lag influence 

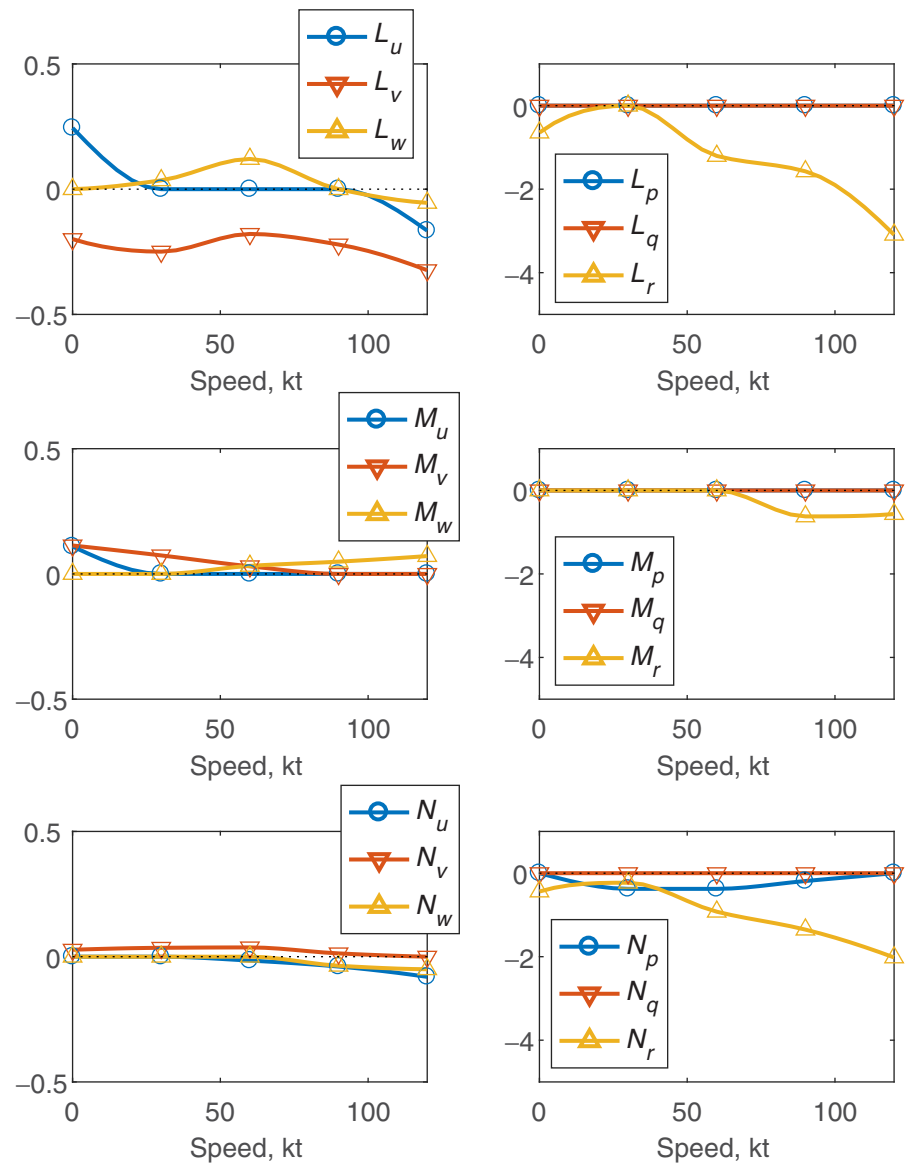

Fig. 13. Identified moment derivatives versus speed.
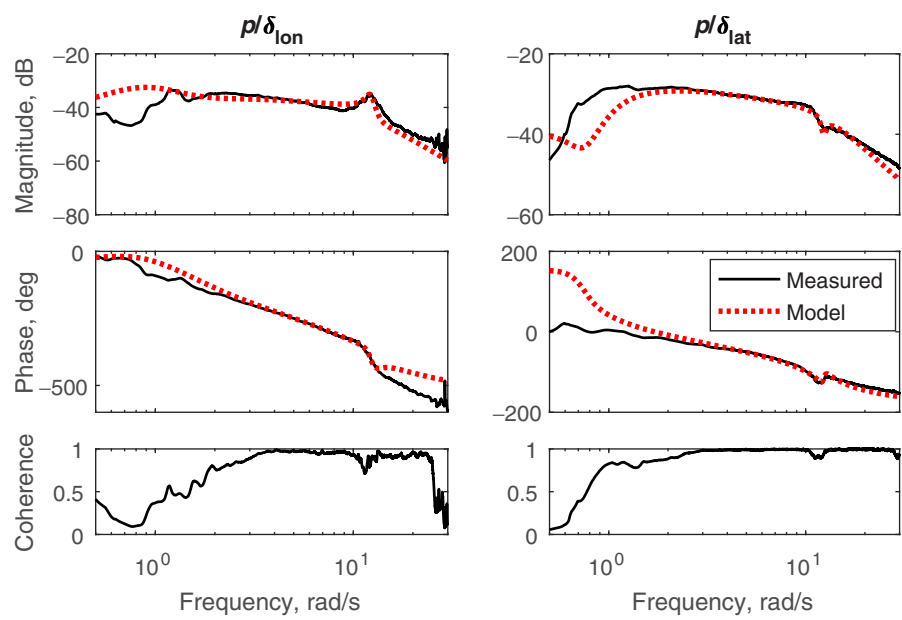

Fig. 14. Match in roll rate due to longitudinal and lateral cyclic inputs (hover).

on the angular rates due to collective input is not significant throughout the flight envelope. For this step, all model parameters determined so far were fixed and only the lead-lag model parameters were identified over a frequency range of $10-15 \mathrm{rad} / \mathrm{s}$. Finally, the identified lead-lag parameters were fixed and the remaining parameters reiterated over the full frequency range.
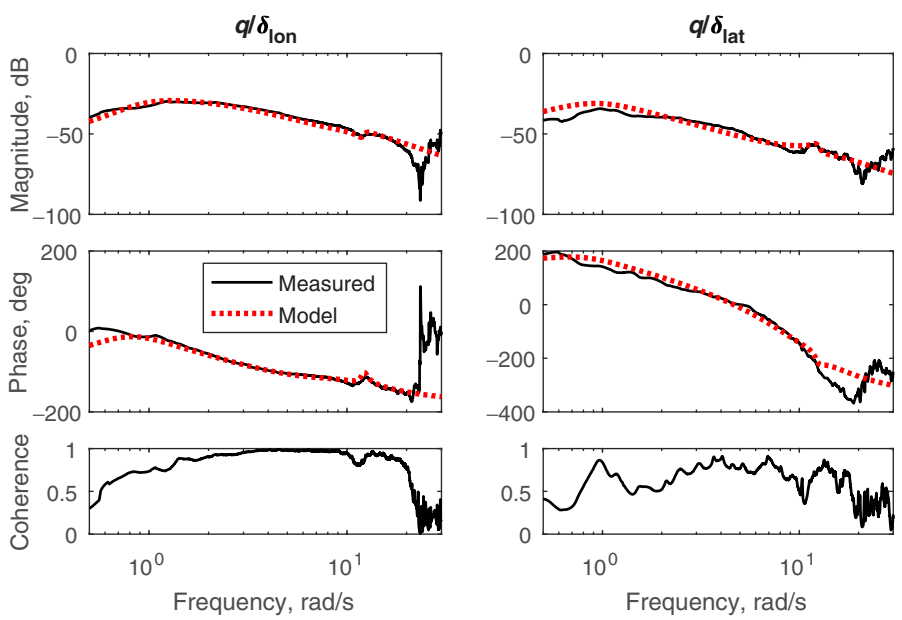

Fig. 15. Match in pitch rate due to longitudinal and lateral cyclic inputs (hover).
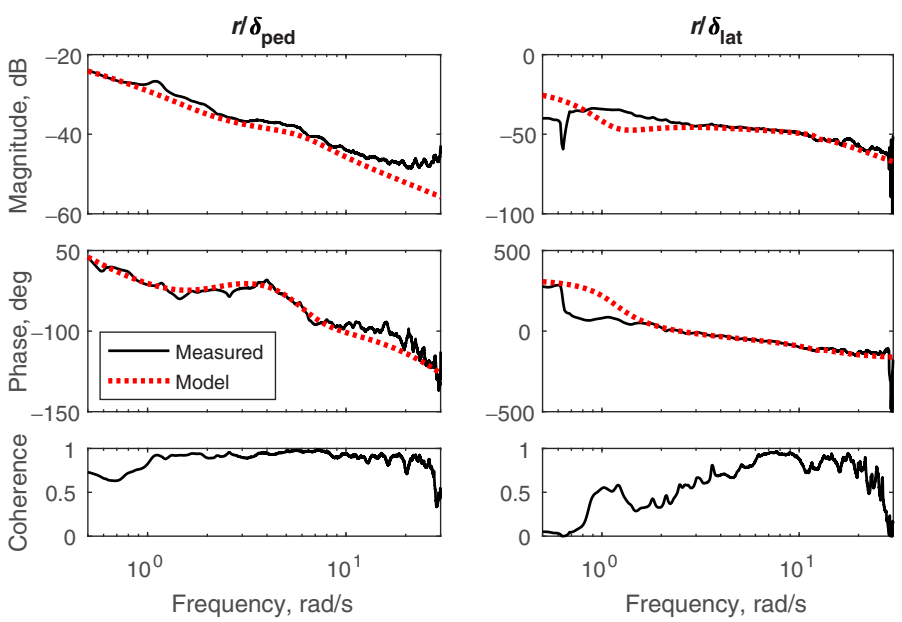

Fig. 16. Match in yaw rate due to pedal and lateral cyclic inputs (hover).

Figures 14-16 illustrate the achieved frequency domain match of the hover model. The models for the forward flight cases are of similar quality.

Some modeling deficits can still be seen where a rising amplitude in the frequency range of $20-30 \mathrm{rad} / \mathrm{s}$ is not covered by the model (see, e.g., $r / \delta_{\text {ped }}$ in Fig. 16). These deficits are attributed to structural modes such as tailboom flexibility and will be accounted for in the future.

Figure 17 shows the achieved time domain match of the velocity components for the 60-kt model.

\section{Conclusions}

System identification was used to generate models for the ACT/FHS helicopter that fulfill the following requirements:

1) The models should be accurate for frequencies up to $30 \mathrm{rad} / \mathrm{s}$.

2) It must be possible to interpolate between the models for the five reference speeds.

3) The models have to be invertible with respect to the output variables $p, q, r, a_{z}$ and thus the corresponding submodels should not have any positive transmission zeros. 


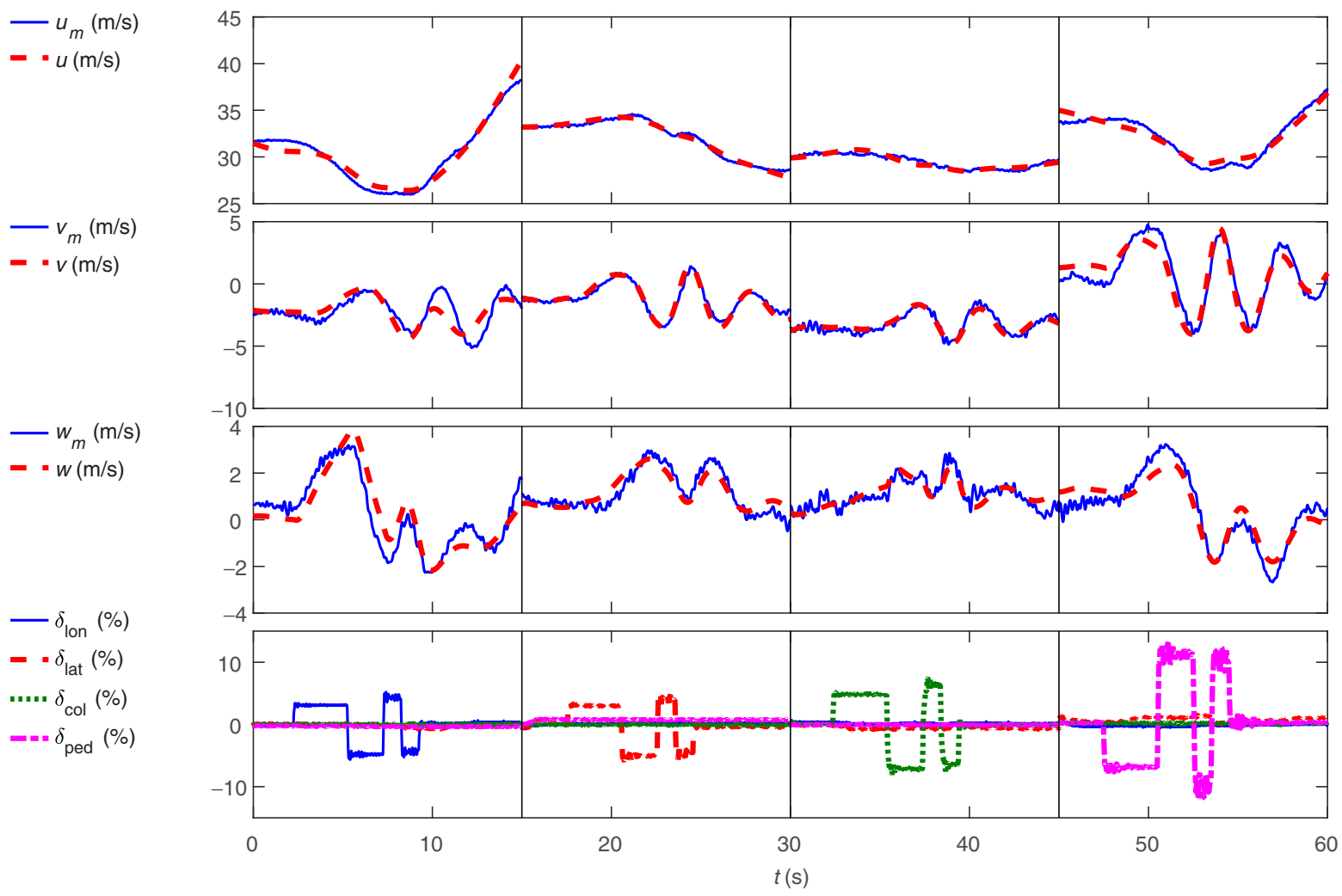

Fig. 17. Time domain match in the velocity components $(60 \mathrm{kt})$.

Different modeling variants for the rotor DOFs and for the engine dynamics were investigated, leading to the following conclusions:

1) Reformulating the explicit flapping formulation so that the flapping angles are also excited by the collective control input yields a model that provides a good separation of rigid-body and rotor parameters, has no positive transmission zeros, and provides a match that is comparable to the implicit flapping formulation.

2) Coning has to be neglected in the modeling of the vertical axis to avoid positive transmission zeros in forward flight. Otherwise, coning should be included to improve the match especially in hover.

3) The new modeling variant for the regressive lead-lag with only one second-order dipole combines the advantages of both of the other two-dipole modeling variants found in the literature and is simpler because it needs only two lead-lag states.

4) Accounting for engine dynamics improves the yaw rate response to both pedal and collective inputs, especially in hover.

Models with this extended structure were identified for five reference speeds and form the basis for a quasi-nonlinear simulation and for control law development.

\section{Appendix: Relationship between One-Dipole and Two-Dipole Formulation}

The relationship between the lead-lag formulation with two dipoles acting on the inputs and the model with only one dipole that is triggered by both the longitudinal and the lateral cyclic input can be derived by comparing the numerator coefficients in the transfer functions from Eqs. (36) and (38).

The coefficients for $s^{2}$ are $L_{\delta_{\text {lon }}}$ and $L_{\delta_{\text {lat }}}$, respectively, $M_{\delta_{\text {lon }}}$ and $M_{\delta_{\text {lat }}}$ for both models, which means that the control derivatives for both models are identical.
Comparing the coefficients for $s^{1}$ and taking into account Eqs. (35) and (39) for the model coefficients yields

$$
\begin{aligned}
L_{x_{2}} & =L_{\delta_{\mathrm{lon}}}\left(2 \zeta_{x p} \omega_{x p}-2 \zeta_{\mathrm{ll}} \omega_{\mathrm{ll}}\right)=L_{z_{2}}+L_{z_{1}} D_{\delta_{\mathrm{lon}}} \\
L_{y_{2}} & =L_{\delta_{\mathrm{lat}}}\left(2 \zeta_{y p} \omega_{y p}-2 \zeta_{11} \omega_{\mathrm{ll}}\right)=L_{z_{2}}+L_{z_{1}} D_{\delta_{\mathrm{lat}}} \\
M_{x_{2}} & =M_{\delta_{\mathrm{lon}}}\left(2 \zeta_{x q} \omega_{x q}-2 \zeta_{\mathrm{ll}} \omega_{\mathrm{ll}}\right)=M_{z_{2}}+M_{z_{1}} D_{\delta_{\mathrm{lon}}} \\
M_{y_{2}} & =M_{\delta_{\mathrm{lat}}}\left(2 \zeta_{y q} \omega_{y q}-2 \zeta_{\mathrm{Il}} \omega_{\mathrm{ll}}\right)=M_{z_{2}}+M_{z_{1}} D_{\delta_{\mathrm{lat}}}
\end{aligned}
$$

Similarly, comparing the coefficients for $s^{0}$ yields

$$
\begin{aligned}
& L_{x_{1}}=L_{\delta_{\text {lon }}}\left(\omega_{x p}^{2}-\omega_{11}^{2}\right)=L_{z_{1}}-L_{z_{2}} D_{\delta_{\text {lon }}} \omega_{1 \mathrm{ll}}^{2}+L_{z_{1}} D_{\delta_{\text {lon }}} 2 \zeta_{\mathrm{ll}} \omega_{\mathrm{ll}} \\
& L_{y_{2}}=L_{\delta_{\text {lat }}}\left(\omega_{y p}^{2}-\omega_{\mathrm{ll}}^{2}\right)=L_{z_{1}}-L_{z_{2}} D_{\delta_{\text {lat }}} \omega_{\mathrm{ll}}^{2}+L_{z_{1}} D_{\delta_{\text {lat }}} 2 \zeta_{\mathrm{ll}} \omega_{\mathrm{ll}} \\
& M_{x_{2}}=M_{\delta_{\mathrm{lon}}}\left(\omega_{x q}^{2}-\omega_{\mathrm{ll}}^{2}\right)=M_{z_{1}}-M_{z_{2}} D_{\delta_{\mathrm{lon}}} \omega_{\mathrm{ll}}^{2}+M_{z_{1}} D_{\delta_{\mathrm{lon}}} 2 \zeta_{\mathrm{ll}} \omega_{\mathrm{ll}} \\
& M_{y_{2}}=M_{\delta_{\mathrm{lat}}}\left(\omega_{y q}^{2}-\omega_{\mathrm{ll}}^{2}\right)=M_{z_{1}}-M_{z_{2}} D_{\delta_{\mathrm{lat}}} \omega_{\mathrm{ll}}^{2}+M_{z_{1}} D_{\delta_{\mathrm{lat}}} 2 \zeta_{\mathrm{ll}} \omega_{\mathrm{ll}}
\end{aligned}
$$

This shows that the coefficients of the two-dipole formulation can be calculated from those for the one-dipole version.

For the reverse relationship, subtracting the first and second equation from (A.1) yields

$$
L_{x_{2}}-L_{y_{2}}=L_{z_{1}}\left(D_{\delta_{\text {lon }}}-D_{\delta_{\text {lat }}}\right)
$$

Multiplying the first equation from (A.1) by $-D_{\delta_{\text {lat }}}$ and adding the second equation multiplied by $D_{\delta_{\text {lon }}}$ gives

$$
D_{\delta_{\text {lon }}} L_{y_{2}}-D_{\delta_{\text {lat }}} L_{x_{2}}=L_{z_{2}}\left(D_{\delta_{\text {lon }}}-D_{\delta_{\text {lat }}}\right)
$$


Similarly, subtracting the first and second equation from (A.2) yields

$$
L_{x_{1}}-L_{y_{1}}=L_{z_{1}}\left(D_{\delta_{\text {lon }}}-D_{\delta_{\text {lat }}}\right) 2 \zeta_{\text {ll }} \omega_{\text {ll }}-L_{z_{2}}\left(D_{\delta_{\text {lon }}}-D_{\delta_{\text {lat }}}\right) \omega_{11}^{2}
$$

and multiplying the first equation from (A.2) by $-D_{\delta_{\text {lat }}}$ and adding the second equation multiplied by $D_{\delta_{\text {lon }}}$ gives

$$
D_{\delta_{\text {lon }}} L_{y_{1}}-D_{\delta_{\text {lat }}} L_{x_{1}}=L_{z_{1}}\left(D_{\delta_{\text {lon }}}-D_{\delta_{\text {lat }}}\right)
$$

Inserting Eqs. (A.3) and (A.4) into Eq. (A.5) yields

$$
L_{x_{1}}-L_{y_{1}}=\left(D_{\delta_{\text {lon }}} L_{y_{2}}-D_{\delta_{\text {lat }}} L_{x_{2}}\right) \omega_{\mathrm{ll}}^{2}+\left(L_{x_{2}}-L_{y_{2}}\right) 2 \zeta_{11} \omega_{\mathrm{ll}}
$$

or

$$
\left(D_{\delta_{\text {lon }}} L_{y_{2}}-D_{\delta_{\text {lat }}} L_{x_{2}}\right) \omega_{\mathrm{ll}}^{2}=L_{x_{1}}-L_{y_{1}}+\left(L_{x_{2}}-L_{y_{2}}\right) 2 \zeta_{11} \omega_{\mathrm{ll}}
$$

and combining Eqs. (A.3) and (A.6) gives

$$
L_{x_{2}}-L_{y_{2}}=D_{\delta_{\text {lon }}} L_{y_{1}}-D_{\delta_{\text {lat }}} L_{x_{1}}
$$

Now that $L_{z_{1}}$ and $L_{z_{2}}$ have been eliminated, $D_{\delta_{\text {lon }}}$ and $D_{\delta_{\text {lat }}}$ can be separated by multiplying Eq. (A.8) by $L_{x_{1}}$ and subtracting Eq. (A.9) multiplied by $L_{x_{2}} \omega_{11}^{2}$

$$
\begin{aligned}
D_{\delta_{\text {lon }}}\left(L_{x_{1}} L_{y_{2}}-L_{x_{2}} L_{y_{1}}\right) \omega_{\mathrm{ll}}^{2}= & L_{x_{1}}\left(L_{x_{1}}-L_{y_{1}}\right)+L_{x_{1}}\left(L_{x_{2}}-L_{y_{2}}\right) 2 \zeta_{\mathrm{ll}} \omega_{\mathrm{ll}} \\
& -L_{x_{2}}\left(L_{x_{2}}-L_{y_{2}}\right) \omega_{\mathrm{ll}}^{2}
\end{aligned}
$$

and multiplying Eq. (A.8) by $L_{y_{1}}$ and subtracting Eq. (A.9) multiplied by $L_{y_{2}}$ yields

$$
\begin{aligned}
D_{\delta_{\text {lat }}}\left(L_{x_{1}} L_{y_{2}}-L_{x_{2}} L_{y_{1}}\right) \omega_{\mathrm{ll}}^{2}= & L_{y_{1}}\left(L_{x_{1}}-L_{y_{1}}\right)+L_{y_{1}}\left(L_{x_{2}}-L_{y_{2}}\right) 2 \zeta_{\mathrm{ll}} \omega_{\mathrm{ll}} \\
& -L_{y_{2}}\left(L_{x_{2}}-L_{y_{2}}\right) \omega_{\mathrm{ll}}^{2}
\end{aligned}
$$

Equations (A.10) and (A.11) allow determination of $D_{\delta_{\text {lon }}}$ and $D_{\delta_{\text {lat }}}$ from the coefficients of the two-dipole model. Inserting the results into Eqs. (A.3) and (A.4) then yields $L_{z_{1}}$ and $L_{z_{2}}$.

So far, only the coefficients of the roll rate transfer functions have been used. Analogously, $D_{\delta_{\text {lon }}}$ and $D_{\delta_{\text {lat }}}$ can also be determined from the coefficients of the pitch equations. This leads to a constraint for the coefficients of the two-dipole formulation. Thus, the two model formulations are not fully equivalent.

\section{References}

${ }^{1}$ von Grünhagen, W., Schönenberg, T., Lantzsch, R., Lusardi, J. A., Lee, D., and Fischer, H., "Handling Qualities Studies into the Interaction between Active Sidestick Parameters and Helicopter Response Types," CEAS Aeronautical Journal, Vol. 5, (1 ), 2014, pp. 13-28.

${ }^{2}$ Greiser, S., Lantzsch, R., Wolfram, J., Wartmann, J., Müllhäuser, M., Lüken, T., Döhler, H.-U., and Peinecke, N., "Results of the Pilot Assistance System 'Assisted Low-Level Flight and Landing on Unprepared Landing Sites' Obtained with the ACT/FHS Research Rotorcraft," Aerospace Science and Technology, Vol. 45, September 2015, pp. 215-227.

${ }^{3}$ Kim, H.-M., Nonnenmacher, D., Götz, J., Weber, P., von Hinüber, E., and Knedlik, S., "Initial Flight Tests of an Automatic Slung Load Control System for the ACT/FHS," CEAS Aeronautical Journal, Vol. 7, (2), 2016, pp. 209-224.

${ }^{4}$ Seher-Weiß, S., and von Grünhagen, W., "EC 135 System Identification for Model Following Control and Turbulence Modeling," CEAS2007-275, First CEAS European Air and Space Conference, Berlin, Germany, September 11-13, 2007, pp. 2439-2447.
${ }^{5}$ Greiser, S., and Seher-Weiß, S., "A Contribution to the Development of a Full Flight Envelope Quasi-nonlinear Helicopter Simulation," CEAS Aeronautical Journal, Vol. 5, (1), 2014, pp. 53-66.

${ }^{6}$ Zivan, L., and Tischler, M. B., "Development of a Full Flight Envelope Helicopter Simulation Using System Identification," Journal of the American Helicopter Society, 55, 022003 (2010).

${ }^{7}$ Tobias, E. L., Tischler, M. B., Berger, T., and Hagerott, S. G., "Full Flight-Envelope Simulation and Piloted Fidelity Assessment of a Business Jet Using a Model Stitching Architecture," AIAA SciTech, Modeling and Simulation Technologies Conference, Kissimmee, FL, January 5-9, 2015.

${ }^{8}$ Mansur, M. H., and Tischler, M. B., "Flight Test Comparison of Alternate Strategies for Multi-Loop Control Law Optimization," AHS 69th Annual Forum, Phoenix, AZ, May 21-23 2013.

${ }^{9}$ Wartmann, J., "Model Validation and Analysis Using Feedforward Control Flight Test Data,' CEAS Aeronautical Journal, Vol. 6, (3 ), 2015, pp. 429-439.

${ }^{10}$ Lunze, J., Regelungstechnik 2-Mehrgrößensysteme, Digitale Regelung, 4th ed., Springer-Verlag, Berlin, Germany, 2006, chapter 2.

${ }^{11}$ Dahleh, M., Dahleh, M. A., and Verghese, G., Poles and zeros of MIMO systems (Chapter 27), Lectures on Dynamic Systems and Control, available at https://ocw.mit.edu/courses/electrical-engineering-and -computer-science/6-241j-dynamic-systems-and-control-spring-2011/ readings/MIT6_241JS11_chap27.pdf, accessed April 1, 2019.

${ }^{12}$ Tischler, M. B., and Remple, R. K., Aircraft and Rotorcraft System Identification: Engineering Methods with Flight-Test Examples, 2nd ed., American Institute of Aeronautics and Astronautics, Inc., Reston, VA, 2012.

${ }^{13}$ Seher-Weiß, S., and von Grünhagen, W., "Comparing Explicit and Implicit Modeling of Rotor Flapping Dynamics for the EC 135," CEAS Aeronautical Journal, Vol. 5, (3), 2014, pp. 319-332.

${ }^{14}$ Seher-Weiß, S., "Comparing Different Approaches for Modeling the Vertical Motion of the EC 135," CEAS Aeronautical Journal, Vol. 6, (3), 2015, pp. 395-406.

${ }^{15}$ Wartmann, J., and Seher-Weiß, S., "Application of the PredictorBased Subspace Identification Method to Rotorcraft System Identification," 39th European Rotorcraft Forum, Moscow, Russia, September 3-6, 2013.

${ }^{16}$ Ivler, C. M., "Development and Comparison of Explicit and Implicit Rotor-State Feedback Control Systems for a Fly-by-Wire UH-60," AHS Rotorcraft Handling Qualities Specialists' Meeting, Huntsville, AL, February 19-20, 2014.

${ }^{17}$ Chen, R. T., and Hindson, W. S., "Influence of Dynamic Inflow on the Helicopter Vertical Response," NASA TM 88327, June 1986.

${ }^{18}$ Tischler, M. B., and Tomashofski, C. A., "Flight Test Identification of SH-2G Flapped-Rotor Helicopter Flight Mechanics Models," Journal of the American Helicopter Society, Vol. 47, (1), 2002, pp. 18-32.

${ }^{19}$ Seher-Weiß, S., and von Grünhagen, W., "Development of EC 135 Turbulence Models via System Identification," Aerospace Science and Technology, Vol. 23, (1), December 2012, pp. 43-52.

${ }^{20}$ Ivler, C. M., and Tischler, M. B., "Case Studies of System Identification Modeling for Flight Control Design," Journal of the American Helicopter Society, 58, 012003 (2013).

${ }^{21}$ Greiser, S., and von Grünhagen, W., "Improving System Identification Results: Combining a Physics-Based Stitched Model with Transfer Function Models Obtained through Inverse Simulation," AHS 72nd Annual Forum, West Palm Beach, FL, May 17-19, 2016.

${ }^{22}$ Basset, P.-M., and Brocard, M., "A Fenestron Model for Improving the Helicopter Yaw Dynamics for Flight Simulation," 30th European Rotorcraft Forum, Marseille, France, September 14-16, 2004. 